\title{
POSITIVE FRAGMENTS OF COALGEBRAIC LOGICS *
}

\author{
ADRIANA BALAN ${ }^{a}$, ALEXANDER KURZ $^{b}$, AND JIŘÍ VELEBIL $^{c}$ \\ ${ }^{a}$ University Politehnica of Bucharest, Romania \\ e-mail address: adriana.balan@mathem.pub.ro \\ ${ }^{b}$ University of Leicester, United Kingdom \\ e-mail address: ak155@mcs.le.ac.uk \\ ${ }^{c}$ Faculty of Electrical Engineering, Czech Technical University in Prague, Czech Republic \\ e-mail address: velebil@math.feld.cvut.cz
}

\begin{abstract}
Positive modal logic was introduced in an influential 1995 paper of Dunn as the positive fragment of standard modal logic. His completeness result consists of an axiomatization that derives all modal formulas that are valid on all Kripke frames and are built only from atomic propositions, conjunction, disjunction, box and diamond.

In this paper, we provide a coalgebraic analysis of this theorem, which not only gives a conceptual proof based on duality theory, but also generalizes Dunn's result from Kripke frames to coalgebras for weak-pullback preserving functors.

To facilitate this analysis we prove a number of category theoretic results on functors on the categories Set of sets and Pos of posets:

Every functor Set $\rightarrow$ Pos has a Pos-enriched left Kan extension Pos $\rightarrow$ Pos. Functors arising in this way are said to have a presentation in discrete arities. In the case that Set $\rightarrow$ Pos is actually Set-valued, we call the corresponding left Kan extension Pos $\rightarrow$ Pos its posetification.
\end{abstract}

A set functor preserves weak pullbacks if and only if its posetification preserves exact squares. A Pos-functor with a presentation in discrete arities preserves surjections.

The inclusion Set $\rightarrow$ Pos is dense. A functor Pos $\rightarrow$ Pos has a presentation in discrete arities if and only if it preserves coinserters of 'truncated nerves of posets'. A functor Pos $\rightarrow$ Pos is a posetification if and only if it preserves coinserters of truncated nerves of posets and discrete posets.

A locally monotone endofunctor of an ordered variety has a presentation by monotone operations and equations if and only if it preserves Pos-enriched sifted colimits.

2012 ACM CCS: [Theory of computation]: Logic.

Key words and phrases: coalgebraic logic, duality, positive modal logic.

* This is an extended and improved version of 9 .

${ }^{a}$ Adriana Balan was supported by CNCSIS project PD-19/03.08.2010.

${ }^{c}$ Jiř́ Velebil is supported by the grant No. P202/11/1632 of the Czech Science Foundation. 


\section{Contents}

1. Introduction

2. A review of coalgebraic logic

3. A review of Pos-enriched categories

4. Presenting functors on Pos

5. Presenting functors on ordered varieties

6. Positive coalgebraic logic

7. Monotone predicate liftings

8. Conclusions

References

\section{InTRODUCTION}

Consider modal logic as given by atomic propositions, Boolean operations, and a unary box, together with its usual axiomatisation stating that box preserves finite meets. In [23], Dunn answered the question of an axiomatisation of the positive fragment of this logic, where the positive fragment is given by atomic propositions, lattice operations, and unary box and diamond (but no negation).

Here we seek to generalize this result from Kripke frames to coalgebras for a weak pullback preserving functor. Whereas Dunn had no need to justify that the positive fragment actually adds a modal operator (the diamond), the general situation requires a conceptual clarification of this step. And, as it turns out, what looks innocent enough in the familiar case is at the heart of the general construction.

In the general case, we start with a functor $T:$ Set $\rightarrow$ Set. From $T$ we can obtain by duality a functor $L: \mathrm{BA} \rightarrow \mathrm{BA}$ on the category $\mathrm{BA}$ of Boolean algebras, so that the free $L$-algebras are exactly the Lindenbaum algebras of the modal logic. We are going to take the functor $L$ itself as the category theoretic counterpart of the corresponding modal logic. How should we construct the positive $T$-logic? Dunn gives us a hint in that he notes that in the same way as standard modal logic is given by algebras over BA, positive modal logic is given by algebras over the category DL of (bounded) distributive lattices. It follows that the positive fragment of (the logic corresponding to) $L$ should be a functor $L^{\prime}: \mathrm{DL} \rightarrow \mathrm{DL}$ which, in turn, by duality, should arise from a functor $T^{\prime}:$ Pos $\rightarrow$ Pos on the category Pos of posets and monotone maps.

The centrepiece of our construction is now the observation that any functor $T:$ Set $\rightarrow$ Set has a universal extension to a functor $T^{\prime}:$ Pos $\rightarrow$ Pos. Theorem 6.20 then shows that this construction $T \mapsto T^{\prime} \mapsto L^{\prime}$ indeed gives the positive fragment of $L$ and so generalizes Dunn's theorem.

An important observation about the positive fragment is the following: given any Boolean formula, we can rewrite it as a positive formula with negation appearing only on atomic 
propositions. In other words, the translation $\beta$ from positive logic to Boolean logic given by

$$
\begin{gathered}
\beta(\diamond \phi)=\neg \square \neg \beta(\phi) \\
\beta(\square \phi)=\square \beta(\phi)
\end{gathered}
$$

induces a bijection (on equivalence classes of formulas taken up to logical equivalence). More algebraically, we can formulate this as follows.

Given a Boolean algebra $B \in \mathrm{BA}$, let $L B$ be the free Boolean algebra generated by the set $\{\square b \mid b \in B\}$ modulo the axioms of modal logic. Given a distributive lattice $A$, let $L^{\prime} A$ be the free distributive lattice generated by $\{\square a \mid a \in A\} \cup\{\triangleright a \mid a \in A\}$ modulo the axioms of positive modal logic. Further, let us denote by $W: \mathrm{BA} \rightarrow \mathrm{DL}$ the forgetful functor. Then the above observation that every modal formula can be written, up to logical equivalence, as a positive modal formula with negations pushed to atoms, can be condensed into the statement that the (natural) distributive lattice homomorphism

$$
\beta_{B}: L^{\prime} W B \rightarrow W L B
$$

induced by Equations (1.1)-(1.2) is an isomorphism.

Our main results, presented in Sections 6 and 7, are the following. If $T^{\prime}$ is an extension of $T$ and $L, L^{\prime}$ are the induced logics, then $\beta: L^{\prime} W \rightarrow W L$ exists. If, moreover, $T^{\prime}$ is the induced extension (posetification) of $T$ and $T$ preserves weak pullbacks, then $\beta$ is an isomorphism (Theorem 6.20). Furthermore, in the same way as the induced logic $L$ can be seen as the logic of all predicate liftings of $T$, the induced logic $L^{\prime}$ is the logic of all monotone predicate liftings of $T$ (Theorem [7.1).

These results depend crucially on the fact that the posetification $T^{\prime}$ of $T$ is defined as a completion with respect to Pos-enriched colimits. We devote Section 4 to establishing some results on posetifications used later. We show that posetifications always exists (Theorem 4.3). Moreover, we characterize those functors Pos $\rightarrow$ Pos that arise as posetifications as the functors that preserve coinserters of 'truncated nerves of posets' and discrete posets (Theorem 4.12). We also establish properties of posetifications needed in Section 6, for example, that a functor Set $\rightarrow$ Set preserves weak pullbacks if and only if its posetification preserves exact squares (Theorem 4.10).

On the algebraic side, the move to Pos-enriched colimits guarantees that the modal operations are monotone. In Section 5, and recalling [43, Theorem 4.7] stating that a functor $L^{\prime}: \mathrm{DL} \rightarrow \mathrm{DL}$ preserves ordinary sifted colimits if and only if it has a presentation by operations and equations, we show that $L^{\prime}: \mathrm{DL} \rightarrow \mathrm{DL}$ preserves enriched sifted colimits if and only if it has a presentation by monotone operations and equations (Theorem 5.16). To see the relevance of a presentation result specific to monotone operations, observe that in the example of positive modal logic it is indeed the case that both $\square$ and $\diamond$ are monotone.

From the point of view of category theory the results of Sections 4 and 5 are of independent interest. In addition to what we already discussed, we introduce the concept of functors Pos $\rightarrow$ Pos with presentations in discrete arities. They generalise posetifications and are functors that arise as left Kan extensions of functors $H$ : Set $\rightarrow$ Pos along the discrete functor $D$ : Set $\rightarrow$ Pos. They are characterised as those functors preserving coinserters of 'truncated nerves of posets' (Theorem 4.13). An important property of Pos-functors with 
presentations in discrete arities is that-like Set-functors but unlike general Pos-functorsthey preserve surjections (Proposition 4.15).

Acknowledgments. The authors would like to thank the referees for their valuable suggestions.

\section{A ReView of COAlgebraic logid}

A Kripke model $(W, R, v)$ (see e.g. [15] for an introduction to modal logic) with $R \subseteq W \times W$ and $v: W \rightarrow 2^{\text {AtProp }}$ can also be described as a coalgebra $W \rightarrow \mathcal{P} W \times 2^{\text {AtProp }}$, where $\mathcal{P} W$ stands for the powerset of $W$. This point of view suggests to generalize modal logic from Kripke frames to coalgebras

$$
\xi: X \rightarrow T X
$$

where $T$ may now be any functor $T:$ Set $\rightarrow$ Set, see 52 for an introduction. We recover Kripke models by putting $T X=\mathcal{P} X \times 2^{\text {AtProp }}$. We also recover the so-called bounded morphisms or p-morphisms as coalgebras morphisms $f:(X, \xi) \rightarrow\left(X^{\prime}, \xi^{\prime}\right)$, that is, as maps $f: X \rightarrow X^{\prime}$ such that $T f \circ \xi=\xi^{\prime} \circ f$.

2.A. Coalgebras and algebras. More generally, for any category $\mathscr{K}$ and any functor $T: \mathscr{K} \rightarrow \mathscr{K}$, we have the category $\operatorname{Coalg}(T)$ of $T$-coalgebras with objects and morphisms as above. Dually, $\operatorname{Alg}(T)$ is the category where the objects $\alpha: T X \rightarrow X$ are arrows in $\mathscr{K}$ and where the morphisms $f:(X, \alpha) \rightarrow\left(X^{\prime}, \alpha^{\prime}\right)$ are arrows $f: X \rightarrow X^{\prime}$ in $\mathscr{K}$ such that $f \circ \alpha=\alpha^{\prime} \circ T f$. It is worth noting that $T$-coalgebras over $\mathscr{K}$ are dual to $T^{\mathrm{op}}$-algebras over $\mathscr{K}^{\mathrm{op}}$, that is, $\operatorname{Coalg}(T)^{\mathrm{op}}=\operatorname{Alg}\left(T^{\mathrm{op}}\right)$. Here $\mathscr{K}^{\mathrm{op}}$ is the category which has the same objects and arrows as $\mathscr{K}$ but domain and codomain of arrows interchanged and $T^{\mathrm{op}}: \mathscr{K}^{\mathrm{op}} \rightarrow \mathscr{K}^{\mathrm{op}}$ is the functor that has the same action on objects and morphisms as $T$.

2.B. Duality of Boolean algebras and sets. The abstract duality between algebras and coalgebras becomes particularly interesting if we put it on top of a concrete duality, such as the dual adjunction between the category Set of sets and functions and the category BA of Boolean algebras. We denote by $P$ : Set ${ }^{\mathrm{op}} \rightarrow \mathrm{BA}$ the functor taking powersets and by $S: \mathrm{BA} \rightarrow$ Set $^{\text {op }}$ the functor taking ultrafilters. Alternatively, we can describe these functors by $P X=\operatorname{Set}(X, 2)$ and $S A=\mathrm{BA}(A, \mathcal{2})$, which also determines their action on arrows (here 2 denotes the two-element Boolean algebra). $P$ and $S$ are adjoint, satisfying $\operatorname{Set}(X, S A) \cong$ $\mathrm{BA}(A, P X)$. Restricting $S$ and $P$ to finite Boolean algebras/sets, this adjunction becomes a dual equivalence [28, $\S$ VI.(2.3)].

2.C. Boolean logics for coalgebras, syntax. What now are logics for coalgebras? We follow a well-established methodology in modal logic [15] and study modal logics via the associated category $\mathscr{A}$ of modal algebras. In modal logic, major milestones are [30] and 24]. In computer science, we have the seminal work on domain theory in logical form [2, 3].

More formally, given a modal logic $\mathcal{L}$ extending Boolean propositional logic with its category $\mathscr{A}$ of modal algebras, we describe $\mathcal{L}$ by a functor

$$
L: \mathrm{BA} \rightarrow \mathrm{BA}
$$


so that the category $\operatorname{Alg}(L)$ of algebras for the functor $L$ coincides with $\mathscr{A}$. In particular, the Lindenbaum algebra of $\mathcal{L}$ will be the initial $L$-algebra.

Example 2.1. Let $L: \mathrm{BA} \rightarrow \mathrm{BA}$ be the functor mapping an algebra $A$ to the algebra $L A$ generated by $\square a, a \in A$, and quotiented by the relation stipulating that $\square$ preserves finite meets, that is,

$$
\square \top=\top \quad \square(a \wedge b)=\square a \wedge \square b
$$

$\operatorname{Alg}(L)$ is the category of modal algebras (Boolean algebras with operators), a result which goes back to [1].

2.D. Boolean logics for coalgebras, semantics. The semantics of such a logic is described by a natural transformation

$$
\delta: L P \rightarrow P T^{\mathrm{op}}
$$

Intuitively, each modal operator in $L P X$ is assigned its meaning as a subset of $T X$. More formally, $\delta$ allows us to lift $P$ : Set ${ }^{\mathrm{op}} \rightarrow$ BA to a functor

$$
\begin{aligned}
\operatorname{Coalg}(T) & \stackrel{P \sharp}{\rightarrow} \operatorname{Alg}(L) \\
(X, \xi) & \mapsto P^{\sharp}(X, \xi)=\left(P X, L P X \stackrel{\delta_{X}}{\longrightarrow} P T X \stackrel{P \xi}{\longrightarrow} P X\right)
\end{aligned}
$$

If we consider a formula $\phi$ to be an element of the initial $L$-algebra (the Lindenbaum algebra of the logic), then the semantics

$$
\llbracket \phi \rrbracket_{(X, \xi)}
$$

of $\phi$ as a subset of a coalgebra $(X, \xi)$ is given by the unique arrow from that initial algebra to $P^{\sharp}(X, \xi)$.

Remark 2.2. This account of the semantics of modal logic is typical for the coalgebraic approach: One first defines a one-step semantics $\delta$ mapping formulas with precisely one layer of modal operators (as described by $L$ ) to one-step-transitions on the semantic side (as described by $T$ ). Then one uses (co)induction to extend the 'one-step situation' to arbitrary formulas and behaviors.

Example 2.3. For the logic $L: \mathrm{BA} \rightarrow \mathrm{BA}$ from Example 2.1 and the powerset functor $\mathcal{P}:$ Set $\rightarrow$ Set we define the (one-step) semantics $\delta_{X}: L P X \rightarrow P^{\text {Pop }} X$ by

$$
\square a \mapsto\{b \subseteq X \mid b \subseteq a\}, \text { for } a \in P X .
$$

It is an old result in domain theory that $\delta_{X}$ is an isomorphism for finite $X$, see [1]. This implies completeness of the axioms (2.1) with respect to Kripke semantics (2.2). 
2.E. Completeness and Expressiveness. Two important properties of logics, with respect to their coalgebraic semantics, that can be discussed parametrically in $T, L$ and the semantics

$$
\delta: L P \rightarrow P T^{\mathrm{op}}
$$

are completeness and expressiveness. The logic given by $(L, \delta)$ is complete if $\llbracket \phi \rrbracket_{(X, \xi)} \subseteq$ $\llbracket \psi \rrbracket_{(X, \xi)}$ for all coalgebras $(X, \xi)$ implies that $\phi \leq \psi$ holds in the initial $L$-algebra. The logic is expressive if for any two elements of a coalgebra $(X, \xi)$ which are not bisimilar, there is a formula that separates them.

In the duality approach to logics as exemplified by work such as Goldblatt's [24] in modal modal logic and Abramsky's [2] in domain theory, completeness and expressiveness follow immediately if $\delta$ is an isomorphism. Since in that work the modal algebras and the coalgebras are based on dually equivalent categories, such as Boolean algebras and Stone spaces (or distributive lattices and Priestley spaces), the requirement that $\delta$ is an isomorphism is reasonable.

In our setting, the situation is different since we only have an adjunction, not an equivalence, relating BA and Set (or DL and Pos). One of the consequences is that $\delta$ typically fails to be an isomorphism and the best we can usually expect is to have $\delta_{X}$ an isomorphism for finite $X$. But, as it turns out, under relatively mild conditions on the functors $T$ and $L$ involved, one can show that the logic $L$ is complete if and only if $\delta$ is injective [40, 42, and that $L$ is expressive if the adjoint transpose $S L \rightarrow T^{\mathrm{op}} S$ of $\delta$ is injective [39, 26] or, in the case of Pos, an embedding [31]. We will pick up this discussion again at the end of Sections 6 and 7 .

2.F. Functors having presentations by operations and equations. Given that the notion of functor is axiomatic and rather abstract, the question arises which functors $L$ : $\mathrm{BA} \rightarrow$ BA can legitimately be considered to be a modal logic. For us, in this paper, the requirement on $L$ is that $L$ has a presentation by operations and equations [18. We will discuss this notion in detail in Section 4, for now it is enough to recall an example.

For a presentation $\left\langle\Sigma_{\mathrm{BA}}, E_{\mathrm{BA}}\right\rangle$ of $\mathrm{BA}$ we let $\Sigma_{\mathrm{BA}}=\{\perp, \top, \neg, \vee, \wedge\}$ and $E_{\mathrm{BA}}$ the usual equations of Boolean algebra. For a presentation $\left\langle\Sigma_{L}, E_{L}\right\rangle$ of the functor $L: \mathrm{BA} \rightarrow \mathrm{BA}$ from Example 2.1 we let $\Sigma_{L}=\{\square\}$ and $E_{L}$ the equations (2.1).

The first reason why the notion of a presentation of a functor is important to us is the following. If $\left\langle\Sigma_{L}, E_{L}\right\rangle$ is a presentation of $L$ by operations $\Sigma_{L}$ and equations $E_{L}$ and if $\left\langle\Sigma_{\mathrm{BA}}, E_{\mathrm{BA}}\right\rangle$ is a presentation of BA then $\operatorname{Alg}(L)$ is presented by

$$
\left\langle\Sigma_{\mathrm{BA}}+\Sigma_{L}, E_{\mathrm{BA}}+E_{L}\right\rangle .
$$

In such a situation, the logical description of $L$-algebra is obtained in a modular way from the logical description of the base category BA and the logical description of the functor $L$. We call the elements of $\Sigma_{L}$ modal operators and the elements of $E_{L}$ modal axioms or just axioms.

The second reason is that the class of functors having presentations can be captured in a purely semantic way: a functor $L$ has a presentation by operations and equations if and only if $L$ is determined by its action on finitely generated free algebras, if and only if $L$ preserves sifted colimits, see [43]. We shall give more details on sifted colimits in Section 3.E below.

Most succinctly, endofunctors of BA having presentations by operations and equations are precisely those that arise as left Kan extensions of their restrictions along the inclusion 
functor $\mathrm{BA}_{f f} \rightarrow \mathrm{BA}$ of the full subcategory $\mathrm{BA}_{f f}$ of $\mathrm{BA}$ consisting of free algebras on finitely many generators.

2.G. The (finitary, Boolean) coalgebraic logic of a Set-functor. The question considered in this paragraph is: given the coalgebra-type $T$, how can we define the logic $\mathbf{L}$ of $T$ ? As there are many different logics $L$ for $T$, we shall use $\mathbf{L}$ for the logic of $T$, that is, the strongest logic that captures all aspects of $T$ that can be expressed by a finitary Boolean logic.

The general considerations laid out above suggest that in order to define the finitary (Boolean) coalgebraic logic associated to a given functor $T$ : Set $\rightarrow$ Set one first puts

$$
\mathbf{L} F n=P T^{\mathrm{op}} S F n
$$

where $F n$ denotes the free Boolean algebra over $n$ generators, for $n$ ranging over natural numbers. The functor $\mathbf{L}: B A \rightarrow B A$ is then defined as a left Kan extension. The semantics $\boldsymbol{\delta}$ is given by observing that natural transformations $\boldsymbol{\delta}: \mathbf{L} P \rightarrow P T^{\mathrm{op}}$ are in bijection with natural transformations

$$
\widehat{\boldsymbol{\delta}}: \mathbf{L} \rightarrow P T^{\mathrm{op}} S
$$

and we can let $\widehat{\boldsymbol{\delta}}$ to be the identity on finitely generated free algebras.

More explicitly, $\mathbf{L} A$ can be represented as the free Boolean algebra on the set

$$
\left\{\sigma\left(a_{1}, \ldots a_{n}\right) \mid \sigma \in P T^{\mathrm{op}} S F n, a_{i} \in A, n<\omega\right\}
$$

modulo appropriate axioms. The semantics $\boldsymbol{\delta}_{X}: \mathbf{L} P X \rightarrow P T^{\mathrm{op}} X$ is given by putting

$$
\boldsymbol{\delta} \sigma\left(a_{1}, \ldots a_{n}\right)=P T^{\mathrm{op}}(\hat{a})(\sigma)
$$

where $\hat{a}: X \rightarrow S F n$ is the adjoint transpose of $\left(a_{1}, \ldots a_{n}\right): n \rightarrow U P X$, with $F \dashv U: \mathrm{BA} \rightarrow$ Set being the familiar adjunction.

It is worth noting that the elements in $P T^{\mathrm{op}} S F n$ are, by the Yoneda Lemma, in one-to-one correspondence with natural transformations $\operatorname{Set}\left(-, 2^{n}\right) \rightarrow \operatorname{Set}(T-, 2)$. The latter natural transformations are also known as predicate liftings [50]. Hence we see that the logic $\mathbf{L}$ coincides with the logic of all predicate liftings of [53], with the difference that the functor $\mathbf{L}$ also incorporates a complete set of axioms. The axioms are important to us as otherwise the natural transformation $\beta$ mentioned in the introduction, see Equation (1.3), might not exist.

Of course, in concrete examples one is often able to obtain much more succinct presentations:

Proposition 2.4. For $T$ the powerset functor, the functor $\mathbf{L}$ defined by Equation (2.3) is isomorphic to the functor $L$ of Example 2.1.

Proof. In analogy with Equation (2.4) , let $\widehat{\delta}: L \rightarrow P T^{\mathrm{op}} S$ be the transpose of $\delta: L P \rightarrow$ $P P^{\circ p}$ as defined in Equation (2.2). We know from Example 2.3 that $\hat{\delta}_{F n}: L F n \rightarrow$ $P T^{\mathrm{op}} S F n=\mathbf{L} F n$ is an isomorphism. But as both $L$ and $\mathbf{L}$ are determined by their action on finitely generated free algebras, this extends to an isomorphisn $L \rightarrow \mathbf{L}$. 
Remark 2.5. The functor $\mathbf{L}$ is universal in the sense that any other finitary Boolean coalgebraic logic $L$ for $T$ is uniquely determined by the natural transformation $L \rightarrow \mathbf{L}$ constructed in the proof above. More formally, we can express this universality as follows: denote by Sift [BA, BA] the category of sifted-colimit-preserving functors from BA to BA and by $I$ the inclusion Sift[BA, BA] $\rightarrow[\mathrm{BA}, \mathrm{BA}]$. Then $\widehat{\boldsymbol{\delta}}: \mathbf{L} \rightarrow P T^{\mathrm{op}} S$ as given in Equation (2.4) is a final object in the comma category $I / P T^{\mathrm{op}} S$.

Proposition 2.4 can then be understood as saying that the logic defined by finality as above has a simple concrete presentation given by Equations (2.1) $-(2.2)$.

2.H. Outlook: Positive coalgebraic logic. It is evident that, at least for some of the developments above, not only the functor $T$, but also the categories Set and BA can be considered to be parameters. Accordingly, one expects that positive coalgebraic logic takes place over the category DL of (bounded) distributive lattices which in turn, is part of an adjunction $P^{\prime}:$ Pos ${ }^{\mathrm{op}} \rightarrow \mathrm{DL}$, taking upsets, and $S^{\prime}: \mathrm{DL} \rightarrow$ Pos $^{\mathrm{op}}$, taking prime filters, or, equivalently, $P^{\prime} X=\operatorname{Pos}(X, \mathcal{2})$ and $S^{\prime} A=\operatorname{DL}(A, \mathcal{2})$ where $\mathcal{2}$ is, as before, the two-element chain (now considered, depending on the context, either as a poset or as a distributive lattice). Consequently, the 'natural semantics' of positive logics is 'ordered Kripke frames', or coalgebras over posets. One side of this argument is formal: coalgebras over Pos are to logics over DL what coalgebras over Set are to logics over BA. Another side of the argument goes as follows: to provide the semantics for a logic without negation, we need to distinguish between sets and their complements. This is most easily done by stipulating that the semantics of formulas is given by upward closed sets with respect to some order, as the complements then are downward closed sets.

Replaying the developments above with Pos and DL instead of Set and BA, we may define a logic for $T^{\prime}$-coalgebras, with $T^{\prime}$ : Pos $\rightarrow$ Pos, to be given by a natural transformation

$$
\delta^{\prime}: \mathbf{L}^{\prime} P^{\prime} \rightarrow P^{\prime} T^{\prime \text { op }}
$$

where $\mathbf{L}^{\prime}: \mathrm{DL} \rightarrow \mathrm{DL}$ is a functor determined by its action on finitely discretely generated free distributive lattices, namely

$$
\mathbf{L}^{\prime} F^{\prime} D n=P^{\prime} T^{\prime \text { op }} S^{\prime} F^{\prime} D n
$$

and $\boldsymbol{\delta}^{\prime}$ is given by its transpose in the same way as in Equation (2.4). Here, $D:$ Set $\rightarrow$ Pos denotes the functor equipping a set with the discrete order, and $F^{\prime}:$ Pos $\rightarrow$ DL is the functor mapping a poset to the free distributive lattice on it.

Example 2.6. Given a poset $X$, a subset $Y \subseteq X$ is called convex if $y \leq y^{\prime} \leq y^{\prime \prime}$ and $y, y^{\prime \prime} \in Y$ imply $y^{\prime} \in Y$. The convex powerset functor $\mathcal{P}^{\prime}:$ Pos $\rightarrow$ Pos maps a poset to the set of its convex subsets, ordered by the Egli-Milner order, and a monotone map to its direct image. Let now $L^{\prime}: \mathrm{DL} \rightarrow \mathrm{DL}$ be the functor mapping a distributive lattice $A$ to the distributive lattice $L^{\prime} A$ generated by $\square a$ and $\diamond a$ for all $a \in A$, and subject to the relations stipulating that $\square$ preserves finite meets, $\diamond$ preserves finite joins, and that the inequalities

$$
\square a \wedge \diamond b \leq \diamond(a \wedge b) \quad \square(a \vee b) \leq \diamond a \vee \square b
$$

hold.

The natural transformation $\delta_{X}^{\prime}: L^{\prime} P^{\prime} X \rightarrow P^{\prime \prime \mathcal{P}^{\prime o p}} X$ is defined by putting

$$
\diamond a \mapsto\{b \subseteq X \mid b \text { is a convex subset of } X \text { and } b \cap a \neq \emptyset\} \text {, for } a \in P^{\prime} X \text {, }
$$

the clause for $\square a$ being the same as in Equation (2.2). 
Remark 2.7. $\operatorname{Alg}\left(L^{\prime}\right)$ is the category of positive modal algebras of Dunn [23. We shall later see in Corollary 5.17 that it is isomorphic to $\operatorname{Alg}\left(\mathbf{L}^{\prime}\right)$. We have again that for a finite poset $X, \delta_{X}^{\prime}$ is an isomorphism, a representation first stated in [28, 29], the connection with modal logic being given by [1, 51, 59] and investigated from a coalgebraic point of view in [49]. As opposed to [49], we take the set-theoretic semantics of modal logic as fundamental. We therefore do not have to use the topological semantics based on Stone or Priestley duality: all we need is contained in the adjunctions $S \dashv P$ : Set ${ }^{\mathrm{op}} \rightarrow \mathrm{BA}$ and $S^{\prime} \dashv P^{\prime}: \mathrm{Pos}^{\mathrm{op}} \rightarrow \mathrm{DL}$.

2.I. Outlook: Coalgebraic logic enriched over Pos. Moving from ordinary categories to categories enriched over Pos plays a major role in this paper. The reason is that enrichment over Pos takes automatically care of the fact that positive modal logics extend the logic of distributive lattices by monotone modal operations. This is crucial from the point of view of our main application, namely positive modal logic.

Throughout the paper, however, we shall encounter many more reasons why to move to an enriched setting. Some of the reasons are the following.

(1) The category Pos is the cocompletion under enriched sifted colimits of the category of finite sets.

(2) The posetification, to be locally monotone, must be defined as an enriched left Kan extension.

(3) Among all functors on Pos, posetifications are characterized by 'coinserters of truncated nerves' where a coinserter is the enriched analogue of a coequalizer.

(4) In the ordered setting, one is frequently interested in definability by inequations $(\leq)$ instead of definability by equations: quotienting by inequations corresponds to taking a coinserter instead of a coequalizer.

(5) Having a presentation by monotone operations and equations in discrete arities is characterized by preservation of enriched sifted colimits.

\section{A REview of Pos-EnRICHed CATEGories}

Below we recall some notions of enriched category theory needed in the sequel. Most of this section is rather technical, but we have decided to include it in order to keep the paper self-contained. However, for more details, we refer the reader to Kelly's monograph [33].

3.A. The category Pos of posets and monotone maps. The category Pos has partial orders (posets) as objects and monotone maps as arrows. Pos is complete and cocomplete (even locally finitely presentable [4]). Limits are computed as in Set, with the order on the limit being the largest relation making the maps in the cocone monotone. Colimits are easiest to compute in two steps. First, colimits in the category of preorders are computed as in Set, with the preorder on the colimit being the smallest one making the maps in the cocone monotone. Second, one quotients the preorder by anti-symmetry in order to obtain a poset (directed colimits, however, are computed as in Set, see [4]). Pos is also cartesian closed, with the internal hom $[X, Y]$ being the poset of monotone maps from $X$ to $Y$, ordered pointwise. 
3.B. Pos-enriched categories. We shall consider categories enriched in Pos. Thus, a Pos-enriched category $\mathscr{K}$ is a category with ordered homsets, such that composition is monotone in both arguments: $g \circ f \leq k \circ h$ whenever $g \leq k$ and $f \leq h$; a Pos-enriched functor $T: \mathscr{K} \rightarrow \mathscr{L}$ is a locally monotone functor, that is, it preserves the order on the homsets: $f \leq g$ implies $T f \leq T g$. A Pos-natural transformation between locally monotone functors is just a natural transformation, the extra condition of enriched naturality being vacuous here. Consequently, a Pos-adjunction between two Pos-categories is just an ordinary adjunction between two locally monotone functors. The category of Pos-enriched functors from $\mathscr{K}$ to $\mathscr{L}$ and natural transformations between them will be denoted by $[\mathscr{K}, \mathscr{L}]$. The opposite category $\mathscr{K}^{\text {op }}$ of $\mathscr{K}$ has just the sense of morphisms reversed, the order on homposets remains unchanged. To avoid overloaded notation, we shall mostly employ the same notation for a Pos-category $\mathscr{K}$ and its underlying ordinary category, unless it is necessary to emphasize the distinction between them. In such case, the ordinary category will be denoted by $\mathscr{K}_{o}$, and we proceed similarly for Pos-functors.

Besides Pos itself, an example of a Pos-enriched category is Set, the category of sets and functions, considered discretely enriched. In the chain

$$
C \dashv D \dashv V: \text { Pos } \rightarrow \text { Set }
$$

of adjunctions between the connected components functor, the discrete functor and the forgetful one, only the adjunction $C \dashv D$ : Set $\rightarrow$ Pos is enriched; in particular the discrete functor $D:$ Set $\rightarrow$ Pos is locally monotone, while the forgetful functor $V:$ Pos $\rightarrow$ Set fails to be so.

Observe also that, due to the discrete enrichment, any functor Set $\rightarrow$ Set is automatically locally monotone.

3.C. Weighted (co)limits; coinserters; Kan-extensions. Recall from [33] that the proper concepts of limits and colimits in enriched category theory are those of weighted (co)limits. Specifically, the colimit of a Pos functor $G: \mathscr{D} \rightarrow \mathscr{K}$ weighted by a Pos-functor $\varphi: \mathscr{D}^{\text {op }} \rightarrow$ Pos is an object $\varphi * G$ in $\mathscr{K}$, together with an isomorphism

$$
\mathscr{K}(\varphi * G, X) \cong\left[\mathscr{D}^{\mathrm{op}}, \operatorname{Pos}\right](\varphi, \mathscr{K}(G-, X))
$$

of posets, natural in $X$ in $\mathscr{K}$.

Dually, a limit of $G: \mathscr{D} \rightarrow \mathscr{K}$ weighted by $\varphi: \mathscr{D} \rightarrow$ Pos is an object $\{\varphi, G\}$ in $\mathscr{K}$, together with an isomorphism

$$
\mathscr{K}(X,\{\varphi, G\}) \cong[\mathscr{D}, \operatorname{Pos}](\varphi, \mathscr{K}(X, G-))
$$

of posets, again natural in $X$ in $\mathscr{K}$.

One example of weighted colimits are copowers that arise from constant weights and diagrams. Specifically, the copower $P \bullet X$ of a poset $P$ in Pos and an object $X$ in $\mathscr{K}$ is characterized by the natural isomorphism

$$
\mathscr{K}(P \bullet X,-) \cong \operatorname{Pos}(P, \mathscr{K}(X,-))
$$

Another example of weighted (co)limit that will later appear in the paper is the (co)inserter: 
Example 3.1. 35] A coinserter is a colimit that has weight $\varphi: \mathscr{D}^{\mathrm{op}} \rightarrow$ Pos, where $\mathscr{D}$ is the category

$$
\longrightarrow \text {. }
$$

The category $\mathscr{D}$ gets mapped by $\varphi$ to the parallel pair

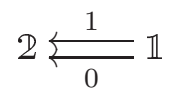

in Pos, with arrow 0 mapping to $0 \in \mathbb{2}$ and arrow 1 mapping to $1 \in \mathbb{Z}$ (recall that $\mathbb{2}$ is the poset $\{0 \leq 1\}$ ). A functor $G$ from $\mathscr{D}$ to a Pos-category $\mathscr{K}$ corresponds to a parallel pair $d^{0}, d^{1}: X \rightrightarrows Y$ of arrows in $\mathscr{K}$.

In more detail, the coinserter of $d^{0}, d^{1}$ consists of an object coins $\left(d^{0}, d^{1}\right)$ and an arrow $c: Y \rightarrow \operatorname{coins}\left(d^{0}, d^{1}\right)$, with $c \circ d^{0} \leq c \circ d^{1}$. The object and the arrow have to satisfy the universal property, of course. Due to the enrichment in posets, the universal property has two aspects:

(1) The 1-dimensional aspect of universality asserts that, given any $q: Y \rightarrow Z$ with $q \circ d^{0} \leq$ $q \circ d^{1}$, there is a unique $h: \operatorname{coins}\left(d^{0}, d^{1}\right) \rightarrow Z$ with $h \circ c=q$.

(2) The 2-dimensional aspect of universality ensures that the above assignment $q \mapsto h$ is monotone. That is, given $q, q^{\prime}: Y \rightarrow Z$ with $q \leq q^{\prime}, q \circ d^{0} \leq q \circ d^{1}$ and $q^{\prime} \circ d^{0} \leq q^{\prime} \circ d^{1}$, the corresponding unique arrows $h, h^{\prime}: \operatorname{coins}\left(d^{0}, d^{1}\right) \rightarrow Z$ satisfy $h \leq h^{\prime}$.

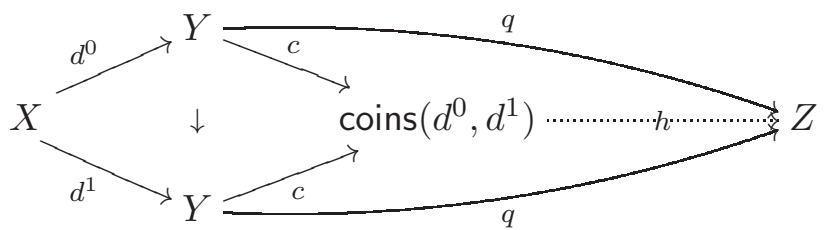

The coinserter is called reflexive if $d^{0}$ and $d^{1}$ have a common right inverse $i: Y \rightarrow X$; that is, if the equalities $d^{0} \circ i=d^{1} \circ i=\operatorname{id}_{Y}$ hold.

By reversing the direction of the arrows, one obtains the dual notion of a (coreflexive) inserter.

We shall later use also co-comma objects, which generalize coinserters, in the sense that this time $\mathscr{D}$ is

and is mapped by $\varphi$ to

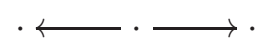

$$
\mathbb{1} \stackrel{0}{\longleftarrow} \stackrel{1}{\longrightarrow} \mathbb{1}
$$

while a functor $G: \mathscr{D} \rightarrow \mathscr{K}$ corresponds to a pair of arrows with common domain $d^{0}: X \rightarrow$ $Y, d^{1}: X \rightarrow Z$. More in detail, the co-comma object of $d^{0}$ and $d^{1}$ consists of an object cocomma $\left(d^{0}, d^{1}\right)$, together with arrows $p: Y \rightarrow \operatorname{cocomma}\left(d^{0}, d^{1}\right), q: Z \rightarrow \operatorname{cocomma}\left(d^{0}, d^{1}\right)$, such that $p \circ d^{0} \leq q \circ d^{1}$, as in the diagram below. We leave to the reader the explicit description of its universal property.

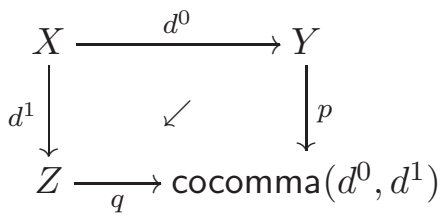


The dual notion is called the comma object.

Remark 3.2. Informally speaking, whereas coequalizers are well-known to take quotients with respect to equivalence relations, coinserters take quotients with respect to preorders.

For later use, we recall how coinserters are built in Pos. For a pair of monotone maps $d^{0}, d^{1}: X \rightarrow Y$, define first a binary relation $\mathbf{r}$ on the underlying set of the poset $Y$ as follows: given $y, y^{\prime} \in Y$, say that $y \mathbf{r} y^{\prime}$ if there are $x_{0}, \ldots, x_{n} \in X$ such that

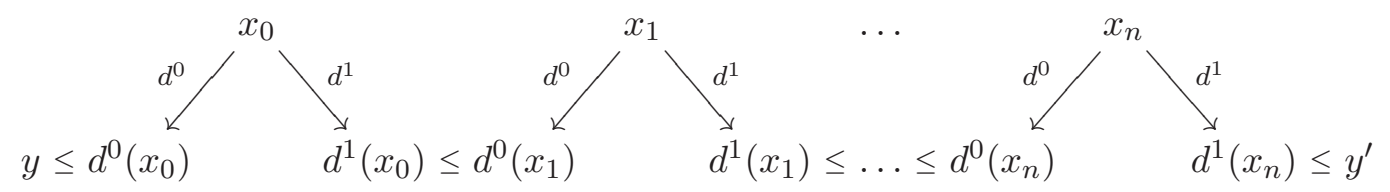

It is easy to see that $\mathbf{r}$ is a reflexive and transitive relation, thus a preorder on $Y$. Then the coinserter of $d^{0}$ and $d^{1}$ is the quotient of $Y$ with respect to the equivalence relation induced by $\mathbf{r}$, with order $[y] \leq\left[y^{\prime}\right]$ if and only if $y \mathbf{r} y^{\prime}$.

Reflexive coinserters play a similar role to reflexive coequalizers in ordinary category theory. For example, one can prove the following Pos-enriched version of [27, Lemma 0.17] (see also [37, Section 2]).

Lemma 3.3. ( $3 \times 3$ lemma for coinserters $)$ Consider in a Pos-category $\mathscr{C}$ the diagram

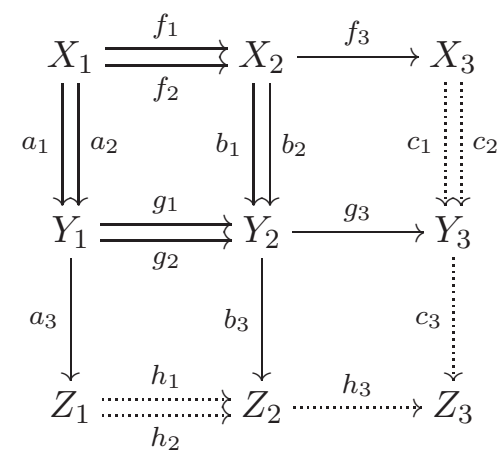

where

(1) The first two rows and columns are coinserters.

(2) The equalities below hold:

$$
b_{i} \circ f_{j}=g_{j} \circ a_{i}(i, j=1,2)
$$

These induce the arrows $c_{1}, c_{2}, h_{1}, h_{2}$ in an obvious way. Finally, let $h_{3}$ be the coinserter (assuming it exists in $\mathscr{C}$ ) of $h_{1}$ and $h_{2}$, and denote by $c_{3}: Y_{3} \rightarrow Z_{3}$ the induced unique mediating arrow. Then:

(1) The last column is also a coinserter.

(2) If additionally the first row and columns are reflexive coinserters, then the diagonal

$$
X_{1} \underset{b_{2} \circ f_{2}}{\stackrel{b_{1} \circ f_{1}}{\longrightarrow}} Y_{2} \stackrel{h_{3} \circ b_{3}}{\longrightarrow} Z_{3}
$$

is again a coinserter, which is reflexive if the second row (column) is again a reflexive coinserter. 
(3) Reflexivity of the first two rows and columns imply reflexivity of the remaining third row and column.

Proof. To see that $c_{3}$ is a coinserter, use first the 2-dimensional aspect of the coinserter $\left(X_{3}, f_{3}\right)$ to conclude $c_{3} \circ c_{1} \leq c_{3} \circ c_{2}$. Next, given $w_{1}: Y_{3} \rightarrow W$ with $w_{1} \circ c_{1} \leq w_{1} \circ c_{2}$, it induces an arrow $w_{2}: Z_{2} \rightarrow W$ such that $w_{2} \circ b_{3}=w_{1} \circ g_{3}$. Then the 2-dimensional part of the coinserter $\left(Z_{1}, a_{3}\right)$ yields $w_{2} \circ h_{1} \leq w_{2} \circ h_{2}$, thus it induces an arrow $w_{3}: Z_{3} \rightarrow W$ with $w_{3} \circ h_{3}=w_{2}$. We have that

$$
w_{3} \circ c_{3} \circ g_{3}=w_{3} \circ h_{3} \circ b_{3}=w_{2} \circ b_{3}=w_{1} \circ g_{3}
$$

and using that $g_{3}$ is an epimorphism we conclude $w_{3} \circ c_{3}=w_{1}$. Finally, if $w_{1}, \bar{w}_{1}: Y_{3} \rightarrow W$ are such that $w_{1} \leq \bar{w}_{1}, w_{1} \circ c_{1} \leq w_{1} \circ c_{2}$ and $\bar{w}_{1} \circ c_{1} \leq \bar{w}_{1} \circ c_{2}$, then successively we obtain $w_{2} \leq \bar{w}_{2}$ and $w_{3} \leq \bar{w}_{2}$ by using the 2 -dimensional aspect of coinserters $\left(Z_{2}, b_{3}\right)$, respectively $\left(Z_{3}, h_{3}\right)$.

For the second part, denote by $i: X_{2} \rightarrow X_{1}$ and $j: Y_{1} \rightarrow X_{1}$ the common right inverses of the parallel pairs of morphisms $f_{1}, f_{2}$, respectively $a_{1}, a_{2}$. Notice then that for an arrow $u_{1}: Y_{2} \rightarrow U$ such that $u_{1} \circ b_{1} \circ f_{1} \leq u_{1} \circ b_{2} \circ f_{2}$, precomposition with $i$ induces the inequality $u_{1} \circ b_{1} \leq u_{1} \circ b_{2}$, thus we can find an arrow $u_{2}: Z_{2} \rightarrow U$ with $u_{2} \circ b_{3}=u_{1}$. In order to see that $u_{2} \circ h_{1} \leq u_{2} \circ h_{2}$, use the first that precomposing

$$
u_{1} \circ g_{1} \circ a_{1}=u_{1} \circ b_{1} \circ f_{1} \leq u_{1} \circ b_{2} \circ f_{2}=u_{1} \circ g_{2} \circ a_{2}
$$

with $j$ yields $u_{1} \circ g_{1} \leq u_{1} \circ g_{2}$, and next use the 2-dimensional aspect of the coinserter $\left(Z_{1}, a_{3}\right)$. From $u_{2} \circ h_{1} \leq u_{2} \circ h_{2}$ we see that there is an arrow $u_{3}: Z_{3} \rightarrow U$ with $u_{3} \circ h_{3}=u_{2}$, thus $u_{3} \circ h_{3} \circ b_{3}=u_{2} \circ b_{3}=u_{1}$. The remaining 2-dimensional aspect of the requested coinserter can be easily proved along these lines, and we leave it to the reader, as well as the assertions on reflexivity.

The importance of reflexive coinserters for us stems from the fact that each poset can be canonically expressed as a reflexive coinserter of discrete posets:

Proposition 3.4. Let $X$ be a poset. Denote by $X_{0}$ its underlying set, and by $X_{1}$ the set of all comparable pairs, $X_{1}=\left\{\left(x, x^{\prime}\right) \in X \mid x \leq x^{\prime}\right\}$. Let $d^{0}, d^{1}: X_{1} \rightarrow X_{0}$ be the maps $d^{0}\left(x, x^{\prime}\right)=x, d^{1}\left(x, x^{\prime}\right)=x^{\prime}$, with common right inverse $i: X_{0} \rightarrow X_{1}, i(x)=(x, x)$. Then the obvious (monotone) map $c: D X_{0} \rightarrow X, c(x)=x$, exhibits $X$ as the coinserter in Pos of the reflexive pair of discrete posets (also called the truncated nerve of the poset)

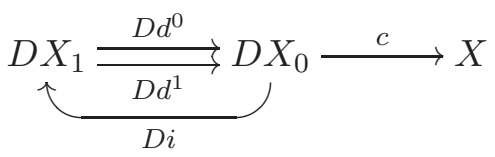

Proof. We leave to the reader to check the straightforward details.

Definition 3.5 (Kan extension). Let $J: \mathscr{A} \rightarrow \mathscr{K}, H: \mathscr{A} \rightarrow \mathscr{L}$ be locally monotone functors. A Pos-enriched left Kan extension of $H$ along $J$, is a locally monotone functor $\operatorname{Lan}_{J} H: \mathscr{K} \rightarrow \mathscr{L}$, such that there is a Pos-natural isomorphism

$$
\left(\operatorname{Lan}_{J} H\right) X \cong \mathscr{K}(J-, X) * H
$$

for each $X$ in $\mathscr{K}$.

\section{Remark 3.6.}


(1) For any locally monotone functor $H^{\prime}: \mathscr{K} \rightarrow \mathscr{L}$, there is an isomorphism

$$
[\mathscr{K}, \mathscr{L}]\left(\operatorname{Lan}_{J} H, H^{\prime}\right) \cong[\mathscr{A}, \mathscr{L}]\left(H, H^{\prime} J\right)
$$

in analogy to the case of unenriched left Kan extensions. In particular, there is a Pos-natural transformation $\alpha: H \rightarrow\left(\operatorname{Lan}_{J} H\right) J$, called unit of the left Kan extension, which is universal in the sense that for a locally monotone functor $H^{\prime}: \mathscr{K} \rightarrow \mathscr{L}$, any Pos-natural transformation $H \rightarrow H^{\prime} J$ factorises through $\alpha$.

In the general enriched setting, requiring the isomorphism (3.1) is strictly weaker than Definition [3.5, but if $\mathscr{L}$ is powered (also called cotensored, see [33, Section 3.7]), it can however be taken as an alternative definition of left Kan extensions (see the discussion after Equation (4.45) in [33]).

(2) Suppose $J: \mathscr{A} \rightarrow \mathscr{K}$ is fully faithful. Then the unit $\alpha: H \rightarrow\left(\operatorname{Lan}_{J} H\right) J$ of the left Kan extension is an isomorphism [33, Proposition 4.23].

(3) By general enriched category theory, the Pos-enriched left Kan extension $\operatorname{Lan}_{J} H$ exists whenever $\mathscr{A}$ is small and $\mathscr{L}$ is cocomplete. But it might exist even when $\mathscr{A}$ is not small, as we shall see later in a special case (Theorem 4.3).

(4) From Equation (3.1) it follows that any locally monotone left adjoint $Q: \mathscr{L} \rightarrow \mathscr{L}^{\prime}$ preserves the Pos-enriched left Kan extension $\operatorname{Lan}_{J} H$, in the sense that the canonical natural transformation

$$
\operatorname{Lan}_{J}(Q H) \rightarrow Q \operatorname{Lan}_{J} H
$$

is an isomorphism.

Example 3.7. Recall that we have denoted by $D \dashv V$ : Pos $\rightarrow$ Set the (ordinary, i.e., not locally monotone) adjunction between the discrete and the forgetful functors, and by $\mathcal{P}:$ Set $\rightarrow$ Set the powerset functor. Then the Pos-enriched left Kan extension of $D \mathcal{P}$ along $D$ is the convex powerset functor (see [57, but also Example 4.5). On the other hand, the ordinary left Kan extension of $D \mathcal{P}$ along $D$ is the composite $D \mathcal{P} V$, which is less interesting, as it maps any poset to the discrete poset of its subsets.

3.D. Ordered varieties. We have seen in Section 2 a close interplay between modal logic and varieties of algebras. The theory of (locally monotone) Pos-functors and their logics of monotone modal operators naturally leads to the world of ordered varieties, as defined by Bloom and Wright in [17].

More precisely, recall that a (finitary) signature $\Sigma$ associates to each natural number $n$ a set of operation symbols $\Sigma_{n}$ of arity $n$. A $\Sigma$-algebra consists of a poset $A$ and, for each $\sigma \in \Sigma_{n}$, a monotone operation $\sigma_{A}: A^{n} \rightarrow A$. An ordered variety is specified by a signature $\Sigma$ and a set of inequations. Bloom [16] proved that varieties are precisely the HSP-closed subclasses of algebras for a signature, provided that we understand closure under $\mathrm{H}$ as the closure under surjective homomorphisms and the closure under $\mathrm{S}$ as closure under embeddings (injective and order-reflecting homomorphisms).

The structure theory of ordered varieties is similar to the one for ordinary varieties. For more details we refer the reader to the original [17] and to the more recent paper [45].

Example 3.8. The category DL of distributive lattices is a variety over Pos if we take algebras to be ordered in the lattice order:

$$
a \leq b \Leftrightarrow a \wedge b=a
$$


The category BA of Boolean algebras is a variety over Pos if we take Boolean algebras to be discretely ordered.

Notice that Boolean algebras can only be discretely ordered, because of the requirement that operations of ordered algebras should be monotone. In the case of Boolean algebras it is not hard to show that the discrete order is the only one that makes all operations (including negation) monotone (see Section 5.C below).

3.E. Sifted weights and sifted colimits; strongly finitary functors. There is a wellknown result that a finitary Set-endofunctor also preserves sifted colimits, or equivalently, filtered colimits and reflexive coequalizers [5, Corollary 6.30]. Below we sketch the corresponding Pos-enriched theory (for more details, we refer to [19, 36, 45, 46]). A weight $\varphi: \mathscr{D}^{\mathrm{op}} \rightarrow$ Pos is called sifted if finite products commute with $\varphi$-colimits in Pos [36]. Equivalently, if the 2-functor $\varphi *-:[\mathscr{D}$, Pos $] \rightarrow$ Pos preserves Pos-enriched finite products. A sifted colimit is a colimit weighted by a sifted weight. Examples of sifted colimits are filtered colimits and reflexive coequalizers, but also reflexive coinserters (see [19]).

There is a close interplay between (ordered) varieties and (enriched) sifted colimits, see also Section 5. For now, remember that in the non-enriched setting, a functor on a variety preserves ordinary sifted colimits if and only if it preserves filtered colimits and reflexive coequalizers 43. In the Pos-enriched setting, a locally monotone functor on an ordered variety preserves (enriched) sifted colimits if and only if it preserves filtered colimits and reflexive coinserters [45, Proposition 6.8].

Let $\operatorname{Set}_{f}$ be the category of finite sets and maps, and let $\iota$ denote the inclusion

$$
\iota \equiv \operatorname{Set}_{f} \stackrel{I}{\hookrightarrow} \text { Set } \stackrel{D}{\rightarrow} \text { Pos }
$$

In [45], following [19, Theorem 8.4], it was noticed that Pos is the free cocompletion of Set $f$ under enriched sifted colimits 1 Briefly, it means that every poset can be expressed as a canonical sifted colimit of finite discrete posets. This colimit can be 'decomposed': every poset is a filtered colimit of finite posets, which in turn arise as reflexive coinserters of discrete finite (po)sets, as explained in Proposition 3.4.

Definition 3.9. ([36]) A strongly finitary functor $T^{\prime}:$ Pos $\rightarrow$ Pos is a locally monotone

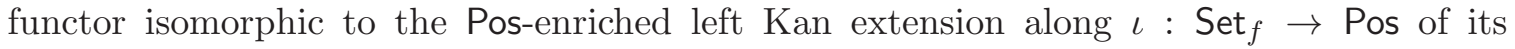
restriction along $\iota$, that is, $T^{\prime} \cong \operatorname{Lan}_{\iota}\left(T^{\prime} \iota\right)$ holds.

Thus, strongly finitary endofunctors of Pos are precisely the locally monotone endofunctors of Pos that preserve (enriched) sifted colimits.

Recall the examples BA and DL of Pos-categories. They are connected by the monadic enriched adjunctions

$$
F \dashv U: \mathrm{BA} \rightarrow \text { Set }, \quad F^{\prime} \dashv U^{\prime}: \mathrm{DL} \rightarrow \text { Pos },
$$

\footnotetext{
${ }^{1}$ In general, let $\Phi$ be a class of weights and let $\mathscr{K}$ be any Pos-category. Following 6 , let $\Phi$-Cocts be the 2-category of $\Phi$-cocomplete categories, $\Phi$-cocontinuous functors, and natural transformations. The free cocompletion of $\mathscr{K}$ with respect to $\Phi$, denoted by $\iota: \mathscr{K} \hookrightarrow \Phi(\mathscr{K})$, is uniquely characterized by the property that composition with $\iota$ induces an equivalence $\Phi$-Cocts $[\Phi(\mathscr{K}), \mathscr{L}] \cong[\mathscr{K}, \mathscr{L}]$ of categories. Its inverse is given by left Kan extension along $\iota$.
} 
where $U$ and $U^{\prime}$ are the corresponding forgetful functors. Extending the notations employed in Section 2.F. we put

$$
\mathbf{J}: \mathrm{BA}_{f f} \rightarrow \mathrm{BA} \quad \text { and } \quad \mathbf{J}^{\prime}: \mathrm{DL}_{f f} \rightarrow \mathrm{DL}
$$

to be the inclusion functors of the full subcategories spanned by the algebras which are free on finite (discrete po)sets.

Lemma 3.10. The inclusion functors $\mathbf{J}$ and $\mathbf{J}^{\prime}$ exhibit $\mathrm{BA}$, respectively $\mathrm{DL}$, as the free enriched cocompletions under sifted colimits of $\mathrm{BA}_{f f}$ and $\mathrm{DL}_{f f}$. In particular, these functors are dense? 2

Proof. We know that the functor $\mathbf{J}: \mathrm{BA}_{f f} \rightarrow \mathrm{BA}$ exhibits $\mathrm{BA}$ as a free cocompletion under sifted colimits (see [43]). Now the conclusion for $\mathbf{J}$ follows because of the discrete enrichment.

For the inclusion functor $\mathbf{J}^{\prime}$ for distributive lattices, the required result is an instance of [45, Theorem 6.10], since DL is a finitary variety of ordered algebras (thus, DL is isomorphic to the category of algebras for a strongly finitary monad on Pos).

Corollary 3.11. A functor $L: B A \rightarrow B A$ has the form $\operatorname{Lan}_{\mathbf{J}}(L \mathbf{J})$ if and only if it preserves (ordinary) sifted colimits. A functor $L^{\prime}: \mathrm{DL} \rightarrow \mathrm{DL}$ has the form $\operatorname{Lan}_{\mathbf{J}^{\prime}}\left(L^{\prime} \mathbf{J}^{\prime}\right)$ if and only if it preserves sifted colimits.

\section{Presenting functors on Pos}

For reasons explained in the introduction, we are interested in the posetifications of functors $T:$ Set $\rightarrow$ Set. Technically, posetifications can be described as enriched left Kan extensions of the functors $D T:$ Set $\rightarrow$ Pos. This suggests to also investigate the more general question of when a left Kan extension of a functor $H$ : Set $\rightarrow$ Pos exists. By general arguments, we know that such a left Kan extension exists if the functor $H$ is finitary, but that would exclude the example $T=\mathcal{P}$ from the introduction. Therefore, in Section 4.A, we show that, in fact, any functor $H$ : Set $\rightarrow$ Pos has an enriched left Kan extension. Then, in Section 4.B. we characterize posetifications among all functors Pos $\rightarrow$ Pos.

4.A. Posetifications and functors Pos $\rightarrow$ Pos with presentations in discrete arities. In order to relate endofunctors of Set and of Pos, we give below an improved version of [8, Definition 1]:

Definition 4.1. Let $T$ be an endofunctor on Set. An endofunctor $T^{\prime}:$ Pos $\rightarrow$ Pos is said to be a Pos-extension of $T$ if $T^{\prime}$ is locally monotone and if the square

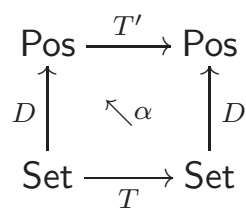

commutes up to a natural isomorphism $\alpha: D T \rightarrow T^{\prime} D$.

\footnotetext{
${ }^{2}$ A general functor $H: \mathscr{A} \rightarrow \mathscr{K}$ is dense if the left Kan extension of $H$ along itself is (naturally isomorphic to) the identity functor on $\mathscr{K}$; that is, each $X$ of $\mathscr{K}$ can be expressed as a canonical colimit $\mathscr{K}(H-, X) * H$ [33, Chapter 5].
} 
A Pos-extension $T^{\prime}$ is called the posetification of $T$ if the above square exhibits $T^{\prime}$ as $\operatorname{Lan}_{D}(D T)$ (in the Pos-enriched sense), having $\alpha$ as its unit.

\section{Remark 4.2.}

(1) Any extension of $T$ has to coincide with $T$ on discrete sets. One would be tempted to take $T^{\prime}=D T V$ as an extension of $T$; but this is not necessarily locally monotone, as $V$ fails to be so. There is also the possibility of choosing $T^{\prime}=D T C$, which does produce an extension, but not the posetification. In fact, $D T C$ is the Pos-enriched right Kan extension $\operatorname{Ran}_{D}(D T)$.

(2) Extensions are not necessarily unique. For example, the identity functor on Pos obviously extends the identity functor on Set, but the same does the functor $D C$ sending a poset to the (discrete) set of its connected components.

(3) In general extensions do not need to inherit all the properties of the Set-functors that they extend. For example, extensions of finitary functors are not necessarily finitary: consider the finitary functor on Set which maps a set $X$ to the set of almost constant sequences on $X$,

$$
T X=\{l: \mathbb{N} \rightarrow X \mid l(n)=l(n+1) \text { for all but a finite number of } n\}
$$

It admits the Pos-extension

$$
T^{\prime}(X, \leq)=\{l:(\mathbb{N}, \leq) \rightarrow(X, \leq) \mid l(n) \leq l(n+1) \text { for all but a finite number of } n\}
$$

mapping a poset $(X, \leq)$ to the poset of almost monotone sequences on $X$, ordered component-wise. But this extension $T^{\prime}$ is not finitary: to see this, consider the family of finite posets $(\{0, \ldots, n-1\})_{n<\omega}$ with the usual order, with inclusion maps, whose colimit in Pos is $(\mathbb{N}, \leq)$. Then one can easily check that $T^{\prime}$ does not preserve the above filtered colimit.

It is clear from general considerations that every finitary endofunctor of Set has a posetification. The point of the next theorem is to drop the finitarity restriction.

Theorem 4.3. Every endofunctor of Set has a posetification.

Proof. The posetification of a functor $T:$ Set $\rightarrow$ Set is constructed as follows. Recall from Proposition 3.4 that each poset $X$ can be expressed as a reflexive coinserter in Pos, as follows:

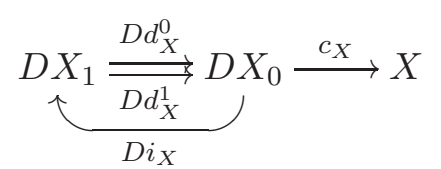

Notice that unlike Proposition 3.4, in the above we have added subscripts to emphasize the poset $X$.

Denote by $e_{X}: D T X_{0} \rightarrow T^{\prime} X$ the coinserter in Pos of the (reflexive) pair $\left(D T d_{X}^{0}, D T d_{X}^{1}\right)$ :

$$
\underbrace{D T X_{1}}_{D T i} \underset{D T d_{X}^{1}}{\stackrel{D T d_{X}^{0}}{\longrightarrow}} D T X_{0} \stackrel{e_{X}}{\longrightarrow} T^{\prime} X
$$

We claim that the assignment $X \mapsto T^{\prime} X$ extends to a locally monotone functor $T^{\prime}:$ Pos $\rightarrow$ Pos, and that $T^{\prime} \cong \operatorname{Lan}_{D}(D T)$ holds.

We proceed in several steps. 
(1) Consider a monotone map $f: X \rightarrow Y$. It induces the obvious maps $f_{0}: X_{0} \rightarrow Y_{0}$ and $f_{1}: X_{1} \rightarrow Y_{1}$. Moreover, the squares
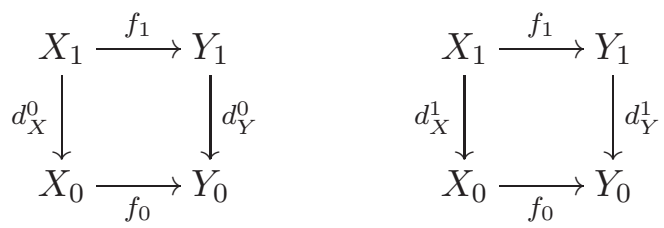

commute. Thus, we have the inequality

$$
e_{Y} \circ D T f_{0} \circ D T d_{X}^{0} \leq e_{Y} \circ D T f_{0} \circ D T d_{X}^{1}
$$

since

$$
D T f_{0} \circ D T d_{X}^{0}=D T d_{Y}^{0} \circ D T f_{1} \quad D T f_{0} \circ D T d_{X}^{1}=D T d_{Y}^{1} \circ D T f_{1}
$$

and

hold.

$$
e_{Y} \circ D T d_{Y}^{0} \leq e_{Y} \circ D T d_{Y}^{1}
$$

Hence one can define $T^{\prime} f: T^{\prime} X \rightarrow T^{\prime} Y$ as the unique mediating monotone map (using the co-universality of the coinserter $e_{X}$ ).

(2) The 1-dimensional aspect of coinserters proves immediately that $T^{\prime}$ preserves composition and identity; that is, $T^{\prime}$ is an ordinary functor Pos $\rightarrow$ Pos.

(3) We show that $T^{\prime}$ is locally monotone; that is, $T^{\prime} f \leq T^{\prime} g$ whenever $f \leq g$ holds, for monotone maps $f, g: X \rightarrow Y$. Observe that $f \leq g$ yields a map $\tau: X_{0} \rightarrow Y_{1}$, $x \mapsto(f(x), g(x))$, such that the triangles
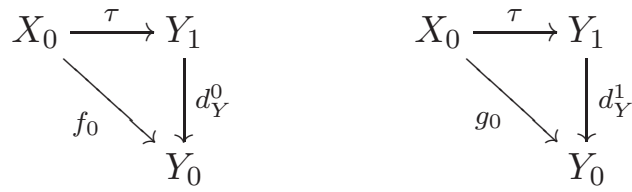

commute.

To prove $T^{\prime} f \leq T^{\prime} g$, it is enough to check that $T^{\prime} f \circ e_{X} \leq T^{\prime} g \circ e_{X}$ holds, for we can then use the 2-dimensional aspect of coinserter $e_{X}$. This inequality follows from

$$
\begin{aligned}
& T^{\prime} f \circ e_{X}=e_{Y} \circ D T f_{0}=e_{Y} \circ D T d_{Y}^{0} \circ D T \tau \\
& T^{\prime} g \circ e_{X}=e_{Y} \circ D T g_{0}=e_{Y} \circ D T d_{Y}^{1} \circ D T \tau,
\end{aligned}
$$

and from the fact that $e_{Y} \circ D T d_{Y}^{0} \leq e_{Y} \circ D T d_{Y}^{1}$ holds.

(4) To prove $T^{\prime} \cong \operatorname{Lan}_{D}(D T)$, we shall show that there is an isomorphism between the poset of natural transformations $T^{\prime} \rightarrow H$ and the poset of natural transformations $D T \rightarrow H D$, for every locally monotone $H:$ Pos $\rightarrow$ Pos (see Remark 3.6(1)).

(a) Consider a natural transformation $\alpha: D T \rightarrow H D$. For every poset $X$, we define $\check{\alpha}_{X}: T^{\prime} X \rightarrow H X$ as the unique mediating map out of a coinserter:

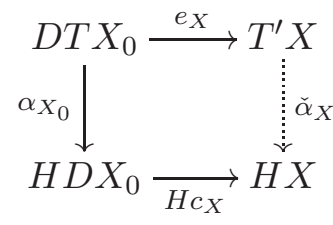

Recall that, above, $c_{X}: D X_{0} \rightarrow X$ is a coinserter of $D d_{X}^{0}, D d_{X}^{1}$. 
The above definition makes sense since

$$
H c_{X} \circ \alpha_{X_{0}} \circ D T d_{X}^{0} \leq H c_{X} \circ \alpha_{X_{0}} \circ D T d_{X}^{1}
$$

holds: the equalities

$$
\alpha_{X_{0}} \circ D T d_{X}^{0}=H D d_{X}^{0} \circ \alpha_{X_{1}} \quad \text { and } \quad \alpha_{X_{0}} \circ D T d_{X}^{1}=H D d_{X}^{1} \circ \alpha_{X_{1}}
$$

follow by naturality, and

$$
c_{X} \circ d_{X}^{0} \leq c_{X} \circ d_{X}^{1}
$$

holds, since $c_{X}$ is a coinserter.

We prove that $\check{\alpha}$ is natural. Consider any monotone map $f: X \rightarrow Y$ and compare

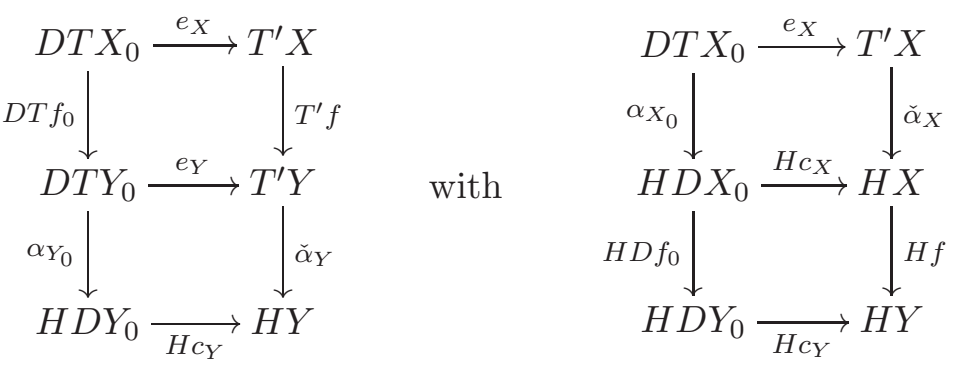

Using naturality of $\alpha$ and co-universality of $e_{X}$, we conclude $H f \circ \check{\alpha}_{X}=\check{\alpha}_{Y} \circ T^{\prime} f$.

(b) Given a natural transformation $\beta: T^{\prime} \rightarrow H$, we define, for every set $X_{0}$, the mapping $\widehat{\beta}_{X_{0}}: D T X_{0} \rightarrow H D X_{0}$ to be $\beta_{D X_{0}}: T^{\prime} D X_{0} \rightarrow H D X_{0}$ (Here we have used the fact that $T^{\prime} D X_{0}$ is naturally isomorphic to $\left.D T X_{0}\right)$.

(c) It is easy then to see that the assignments $\alpha \mapsto \check{\alpha}$ and $\beta \mapsto \widehat{\beta}$ are monotone and inverse to each other.

As a corollary of the proof of the above theorem (replace $D T$ by $H$ everywhere in the above proof) we obtain

Corollary 4.4. For every functor $H$ : Set $\rightarrow$ Pos, the Pos-enriched left Kan extension $\operatorname{Lan}_{D} H:$ Pos $\rightarrow$ Pos exists.

The following gives an example where the construction of posetification as given by Equation (4.2) can be obtained straightforwardly.

Example 4.5. Let $T:$ Set $\rightarrow$ Set be the covariant powerset functor $\mathcal{P}$ and let $X$ be a poset. We shall see how to use Equation (4.2) to determine $\mathcal{P}^{\prime} X$. Taking into account that $D \mathcal{P} d_{X}^{0}$ and $D \mathcal{P} d_{X}^{1}$ are the direct images of the projections, the coinserter (4.2) becomes

$$
D \mathcal{P} X_{1} \underset{D \mathcal{P} d_{X}^{1}}{\stackrel{D \mathcal{P} d_{X}^{0}}{\longrightarrow}} D \mathcal{P} X_{0} \stackrel{e_{X}}{\longrightarrow} \mathcal{P}^{\prime} X
$$

Recall from Remark 3.2 how coinserters are built in Pos: first, consider the relation $\mathbf{r}$ on $\mathcal{P} X_{0}$ given by:

$$
Y \mathbf{r} Z \quad \Leftrightarrow \quad \exists S \subseteq X_{1} \cdot \mathcal{P} d_{X}^{0}(S)=Y \quad \text { and } \quad \mathcal{P} d_{X}^{1}(S)=Z
$$

for subsets $Y, Z \subseteq X_{0}$ (in fact, in Remark 3.2 we have considered the transitive closure of relation (4.3) ). Unravelling the above, we obtain that $Y \mathbf{r} Z$ if and only if

$$
\forall y \in Y . \exists z \in Z . y \leq z \quad \text { and } \quad \forall z \in Z . \exists y \in Y . y \leq z
$$


The relation $\mathbf{r}$ determined by Equation (4.4) is known as the Egli-Milner order: it is reflexive and transitive (consequently, it coincides with its transitive closure), and two subsets $Y, Z$ of $X_{0}$ are equivalent with respect to $\mathbf{r}$ if and only if they have the same convex closure. Thus $\mathcal{P}^{\prime} X$, obtained by quotienting $\mathcal{P} X_{0}$ with respect to the equivalence relation induced by $\mathbf{r}$, is the set of convex subsets of $X$ ordered by the Egli-Milner order.

Similarly, if we let $H$ : Set $\rightarrow$ Pos with $H X=(\mathcal{P} X, \subseteq)$, we obtain the downset functor $\mathcal{D}:$ Pos $\rightarrow$ Pos with $\mathcal{D} X$ ordered by inclusion as the left Kan extension of $H$ along $D$. Dually, if $H$ : Set $\rightarrow$ Pos is given by $H X=(\mathcal{P} X, \supseteq)$, we have that $\operatorname{Lan}_{D} H$ is the upset functor $\mathcal{U}:$ Pos $\rightarrow$ Pos with $\mathcal{U} X$ ordered by reverse inclusion. To verify this, note that two subsets are equivalent according to the left-hand side of (4.4) if and only if they have the same downset closure and two subsets are equivalent according to the right-hand side if and only if they have the same upset closure.

\section{Remark 4.6 (On presentations by monotone operations and equations in discrete arities).}

(1) The posetification built in Theorem 4.3 coincides with the one from [8, (3.2)] given by the coequalizer in Pos

$$
\coprod_{m, n<\omega} \operatorname{Set}(m, n) \times T m \times[D n, X] \rightrightarrows \coprod_{n<\omega} T n \times[D n, X] \rightarrow \operatorname{Lan}_{D}(D T)(X)
$$

if $T$ is finitary (this follows from the fact that $\operatorname{Lan}_{D}(D T) \cong \operatorname{Lan}_{D I}(D T I)$, where remember from Section 3.E that $I:$ Set $_{f} \rightarrow$ Set denotes the inclusion functor).

(2) Let us explain how Equation (4.5) gives a presentation by monotone operations and equations in discrete arities. The operations of arity $n$ are given by $T n$. They are necessarily monotone because the arguments $[D n, X]$ form a poset and we take the coequalizer in Pos. The arities are discrete because $m, n$ range over sets, not posets. For each pair $(m, n)$, we have a poset of equations $\operatorname{Set}(m, n) \times T m \times[D n, X]$ (where the order on the equations does not play a role in the computation of the coequalizer).

(3) For an explicit example of such a presentation by operations and equations, consider $T$ to be the finite powerset functor. First, recall that it can be presented in Set as the quotient of $\coprod_{n<\omega} X^{n}$ by a set of equations specifying that the order and the multiplicity in which elements of the set $X$ occur in lists in $X^{n}$ does not matter. Second, with $X$ now standing for a poset, note that according to [8, Proposition 5], we obtain the posetification of $T$ by quotienting $\coprod_{n<\omega}[D n, X]$ in Pos by the same equations. It is not difficult to show that this gives us the (finite) convex powerset functor on Pos [57, Proposition 5.1].

(4) If we generalize from the posetification of a finitary functor Set $\rightarrow$ Set to the left Kan extension of a finitary functor Set $\rightarrow$ Pos the formula (4.5) is still available and we obtain the same presentations as in item (2), just that the $T n$ need not be discrete anymore. For example, if we let the $T n$ in Equation (4.5) be $\mathcal{P}(n)$ ordered by inclusion, we get a presentation of the functor Pos $\rightarrow$ Pos mapping a poset $X$ to the set of finitely generated downsets ordered by inclusion (Hoare powerdomain).

(5) If we generalize further, giving up that the functor be finitary, we lose the formula (4.5) since the large coproducts may not exist in Pos. Nevertheless, we can still interpret a functor $T$ : Set $\rightarrow$ Pos as having a presentation by monotone operations and equations in discrete arities. This time the arities range over all cardinals, so that for each cardinal $\aleph$ we have a poset of operations $T(\aleph)$ and for each pair $\left(\aleph, \aleph^{\prime}\right)$ of cardinals we have a set of equations $\operatorname{Set}\left(\aleph, \aleph^{\prime}\right) \times T(\aleph) \times\left[D\left(\aleph^{\prime}\right), X\right]$. 
We summarize this discussion by making the following definition.

Definition 4.7. We say that a functor $T^{\prime}:$ Pos $\rightarrow$ Pos has a presentation in discrete arities if $T^{\prime}=\operatorname{Lan}_{D} H$ for some functor $H:$ Set $\rightarrow$ Pos.

The posetification of a functor $T:$ Set $\rightarrow$ Set has been defined by quotienting with respect to the relation given by applying $T$ to the nerve of a poset. Not surprisingly, this construction is closely related to the notion of relation lifting of the functor $T$ :

\section{Remark 4.8 (On posetifications and relation lifting).}

(1) Let $T:$ Set $\rightarrow$ Set be an arbitrary functor. For a relation $\mathbf{r} \subseteq X \times Y$, recall that the $T$-relation lifting of $\mathbf{r}$ is given by the epi-mono factorisation as on the right of the following diagram (see for example [10, 21, 54, 56]):
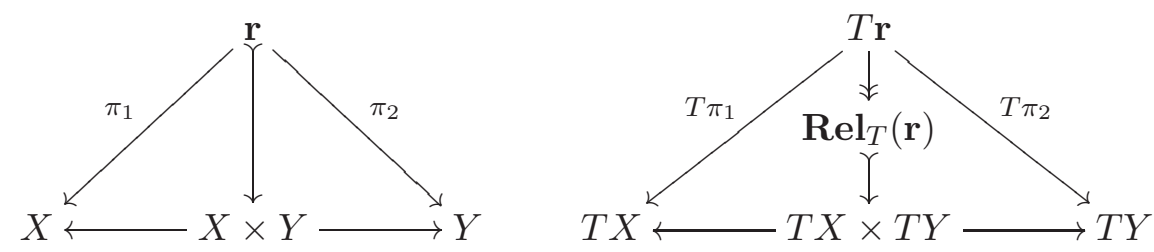

Explicitly,

$$
\operatorname{Rel}_{T}(\mathbf{r})=\left\{(u, v) \in T X \times T Y \mid \exists w \in T \mathbf{r} . T \pi_{1}(w)=u \wedge T \pi_{2}(w)=v\right\}
$$

The relation lifting satisfies the following properties:

(a) It preserves the equality relation: $=_{T X}=\operatorname{Rel}_{T}\left(=_{X}\right)$.

(b) It preserves the inclusion of relations: if $\mathbf{r} \subseteq \mathbf{s}$, then $\operatorname{Rel}_{T}(\mathbf{r}) \subseteq \operatorname{Rel}_{T}(\mathbf{s})$.

(c) If $\mathbf{r} \subseteq X \times Y$ and $\mathbf{s} \subseteq Y \times Z$, then

$$
\operatorname{Rel}_{T}(\mathbf{s} \circ \mathbf{r}) \subseteq \operatorname{Rel}_{T}(\mathbf{s}) \circ \operatorname{Rel}_{T}(\mathbf{r})
$$

with equality if and only if $T$ preserves weak pullbacks.

(d) It preserves converses of relations: $\operatorname{Rel}_{T}\left(\mathbf{r}^{\mathrm{op}}\right)=\mathbf{R e l}_{T}(\mathbf{r})^{\mathrm{op}}$.

(e) Given functions $f: X \rightarrow X^{\prime}, g: Y \rightarrow Y^{\prime}$ and relation $\mathbf{r}^{\prime} \subseteq X^{\prime} \times Y^{\prime}$, then

$$
\operatorname{Rel}_{T}\left((f \times g)^{-1}\left(\mathbf{r}^{\prime}\right)\right) \subseteq(T f \times T g)^{-1}\left(\operatorname{Rel}_{T}\left(\mathbf{r}^{\prime}\right)\right)
$$

with equality if $T$ preserves weak pullbacks.

(2) In addition to the above, we should also mention the (less-known?) fact that relation lifting commutes with functor composition, in the sense that

$$
\operatorname{Rel}_{T S}(\mathbf{r})=\operatorname{Rel}_{T}\left(\operatorname{Rel}_{S}(\mathbf{r})\right)
$$

for any relation $\mathbf{r} \subseteq X \times Y$ and any endofunctors $T, S$ of Set (see [21, Section 4.4], and use that, assuming the axiom of choice, any endofunctor of Set preserves (strong) epimorphisms, i.e., surjective maps).

(3) Recall again that the posetification $T^{\prime}$ of a Set-endofunctor $T$ was obtained via coinserters,

$$
D T X_{1} \underset{D T d^{1}}{\stackrel{D T d^{0}}{\longrightarrow}} D T X_{0} \stackrel{e}{\longrightarrow} T^{\prime} X
$$

for any poset $X$. Observe in fact that the relation $\mathbf{r}$ described in Remark 3.2 at the first stage of the coinserter construction, for the pair of (monotone) maps $D T d^{0}$ and $D T d^{1}$, is precisely the transitive closure of the $T$-relation lifting $\operatorname{Rel}_{T}\left(X_{1}\right)$ of the order 
$X_{1}$ on $X$. By (1)(a) above, $\operatorname{Rel}_{T}\left(X_{1}\right)$ is reflexive, and by (1)(c) it is also transitive if $T$ preserves weak pullbacks. If this is the case, then the posetification $T^{\prime}$ can be explicitly described as mapping a poset $X$ to the quotient poset of the preordered set $\left(T X_{0}, \operatorname{Rel}_{T}\left(X_{1}\right)\right)$.

(4) The above two items provide a proof that, for $T, S$, endofunctors of Set preserving weak pullbacks, the isomorphism

$$
(T S)^{\prime} \cong T^{\prime} S^{\prime}
$$

holds for their posetifications.

The property of a functor $T:$ Set $\rightarrow$ Set preserving weak pullbacks plays an important role in the theory of coalgebras [52. In the category Pos, the following concept is the enriched analogue of a weak pullback.

Definition 4.9 (25]). An exact square in the category Pos of posets, or in the category Preord of preorders, is a diagram

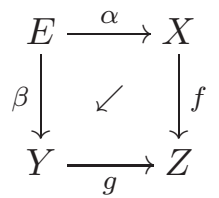

with $f \circ \alpha \leq g \circ \beta$, such that

$$
\forall x \in X, y \in Y . f(x) \leq g(y) \Rightarrow \exists w \in E .(x \leq \alpha(w) \wedge \beta(w) \leq y)
$$

That the above concept generalises weak pullbacks in sets is seen as follows. An exact square of discrete posets is precisely a weak pullback of their underlying sets. Equivalently, the discrete functor $D:$ Set $\rightarrow$ Pos maps weak pullbacks to exact squares and reflects exact squares to weak pullbacks.

Given an endofunctor $T$ of Set, we shall now connect the property of preserving weak pullbacks with the preservation of exact squares by the corresponding posetification $T^{\prime}$. This fact will be used in Theorem 6.20 below.

Theorem 4.10. Let $T$ be any endofunctor of Set and let $T^{\prime}$ be its posetification. Then $T$ preserves weak pullbacks if and only if $T^{\prime}$ preserves exact squares.

Proof. This was proved in [8] under the additional assumption that $T$ is finitary. Here, we present an argument valid for all Set-functors.

We start with the easy implication. Assume $T^{\prime}$ preserves exact squares and consider a weak pullback in Set

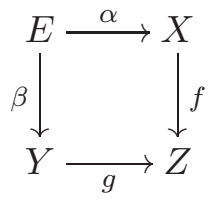


Then Equation (4.7) is mapped by $D$ to an exact square in Pos, and $T^{\prime}$ preserves such by hypothesis. Using the isomorphism $D T \cong T^{\prime} D$, we conclude that

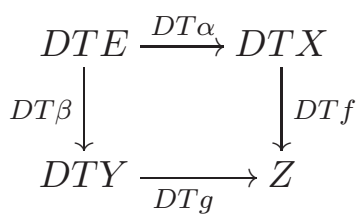

is an exact square of discrete posets, that is, a weak pullback in Set.

Now, we assume that $T$ preserves weak pullbacks and we show that its posetification $T^{\prime}$ preserves exact squares. Recall from Remark 4.8(3) that $T^{\prime} X$ is the quotient of the preordered set $\left(T X_{0}, \operatorname{Rel}_{T}\left(X_{1}\right)\right)$ since $T$ is assumed to preserve weak pullbacks.

In fact, it is easy to see that for each preordered set (poset) $X$, the construct

$$
\bar{T} X=\left(T X_{0}, \operatorname{Rel}_{T}\left(X_{1}\right)\right)
$$

yields a locally monotone functor $\bar{T}$ on the category Preord of preordered sets and monotone mappings.

The inclusion functor Incl : Pos $\rightarrow$ Preord and its left adjoint, the quotient functor Quot : Preord $\rightarrow$ Pos both preserve exact squares [12, Example 6.2], and the composite

$$
\text { Pos } \stackrel{\text { Incl }}{\longrightarrow} \text { Preord } \stackrel{\bar{T}}{\longrightarrow} \text { Preord } \stackrel{\text { Quot }}{\longrightarrow} \text { Pos }
$$

is precisely $T^{\prime}$. Consequently, it is enough to show that $\bar{T}$ preserves exact squares.

Consider thus an exact square in Preord:

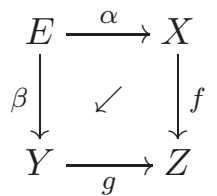

and follow the steps below:

(1) First, the inequality $\bar{T}(f) \circ \bar{T}(\alpha) \leq \bar{T}(g) \circ \bar{T}(\beta)$ holds since $\bar{T}$ is locally monotone.

(2) Next, we form the three pullbacks in Set of the first diagram below, which by hypothesis will be mapped by $T$ to weak pullbacks. As in Proposition 3.4 here $d^{0}, d^{1}: Z_{1} \rightarrow Z$ stand for the projections from the set of comparable pairs to (the underlying set of) $Z$.

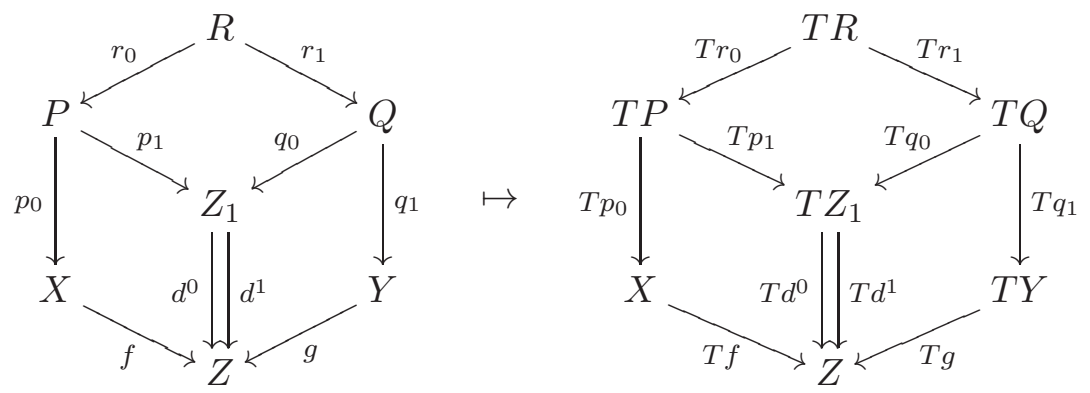


Explicitly,

$$
\begin{aligned}
P & =\{(x, z) \in X \times Z \mid f(x) \leq z\} \\
Q & =\{(z, y) \in Z \times Y \mid z \leq g(y)\} \\
R & =\{(x, y) \in X \times Y \mid f(x) \leq g(y)\} \\
r_{0}(x, y) & =(x, g(y)) \\
r_{1}(x, y) & =(f(x), y)
\end{aligned}
$$

(3) From the description of $R$ above, notice that $R$ is non-empty (as we started from an exact square), and that given $(x, y) \in R$, there is some $w \in E$ such that $x \leq \alpha(w)$ and $\beta(w) \leq y$. Assuming the axiom of choice, fix such a $w \in E$ for each $(x, y) \in R$ and define a map $\theta: R \rightarrow E$ by $\theta(x, y)=w$. It can be considered monotone if $R$ is taken to be a discrete poset.

(4) Consider the cube below in Preord, where $R, P, Q$ carry the discrete (pre)order.

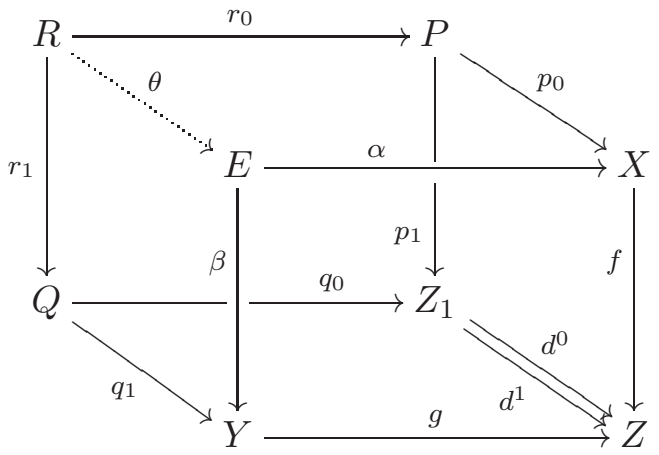

The back, right-hand and bottom faces commute by Equation (4.9). The front face is the exact square of Equation (4.8), in particular $f \circ \alpha \leq g \circ \beta$ holds. The remaining top and left-hand faces commute laxly, in the sense that following inequalities hold:

$$
p_{0} \circ r_{0} \leq \alpha \circ \theta \quad \text { and } \quad \beta \circ \theta \leq q_{1} \circ r_{1}
$$

(5) We are now able to show that $\bar{T}$ preserves exact squares. To that end, let $u \in \bar{T}(X)$, $v \in \bar{T}(Y)$ such that

$$
\bar{T}(f)(u) \leq \bar{T}(g)(v)
$$

in $\bar{T}(Z)=\left(T Z, \operatorname{Rel}_{T}\left(Z_{1}\right)\right)$. That is, $u \in T X, v \in T Y$ and there exists some $w \in T\left(Z_{1}\right)$ such that $T d^{0}(w)=T f(u)$ and $T d^{1}(w)=T g(v)$.

As all the squares in the second diagram in Equation (4.9) are weak pullbacks, we can conclude that there is some $\bar{w} \in T R$ which is mapped to $u \in T X$, respectively $v \in T Y$. Let $\omega=T \theta(\bar{w}) \in T E$. Then one can easily check using Equation (4.10) that $u \leq T \alpha(\omega)$ and $T \beta(\omega) \leq v$ hold.

All in one, we have showed that $\bar{T}$ maps an exact square to an exact square. Thus also the posetification $T^{\prime}$ of $T$ preserves exact squares.

\section{Example 4.11.}

(1) Let $T=$ Id on Set. Then its posetification is the identity functor on posets (recall that the discrete-poset functor $D$ is dense, see the last paragraph in Section 2 of [20]).

(2) If we take $T=\mathcal{P}_{f}$ to be the (finite) power-set functor, then its posetification is the (finitely generated) convex power-set functor, with the Egli-Milner order (see Example 4.5, but also [8, 57]). 
(3) The collection of (finitary) Kripke polynomial endofunctors of Set is inductively defined as follows: $T::=\mathrm{id}\left|T_{X_{0}}\right| T_{0}+T_{1}\left|T_{0} \times T_{1}\right| T^{A} \mid \mathcal{P}_{f}$, where $T_{X_{0}}$ denotes the constant functor to the set $X_{0} ; T_{0}+T_{1}$ is the pointwise coproduct $X \mapsto T_{0} X+T_{1} X ; T_{0} \times T_{1}$ is the pointwise product $X \mapsto T_{0} X \times T_{1} X$; and $T^{A}$ denotes the pointwise exponent functor $X \mapsto(T X)^{A}$, with the set $A$ being finite.

We have just mentioned above that the posetification of the identity functor is again the identity, while for the constant functor $T_{X_{0}}$ it is an easy exercise to check that the posetification is again a constant functor, this time to the discrete poset $D X_{0}$; the posetification of the coproduct $T_{0}+T_{1}$ maps a poset $X$ to the coproduct (in the category of posets) $T_{0}^{\prime} X+T_{1}^{\prime} X$, where $T_{0}^{\prime}$ and $T_{1}^{\prime}$ denote the posetifications of $T_{0}$, respectively $T_{1}$; and similarly for the product functors. Finally, the posetification of the exponent functor $T^{A}$ is $T^{\prime D A}$, where $T^{\prime}$ stands for the posetification of $T$.

(4) Consider now the finitary probability functor Prob : Set $\rightarrow$ Set, given on objects by

$$
\operatorname{Prob}(X)=\left\{p: X \rightarrow[0,1] \mid \sum_{x \in X} p(x)=1, \operatorname{supp}(p)<\infty\right\},
$$

where $\operatorname{supp}(p)=\{x \in X \mid p(x) \neq 0\}$, and by

$$
\operatorname{Prob}(f)(p)(y)=\sum_{y=f(x)} p(x), \text { for a function } f: X \rightarrow Y .
$$

on morphisms. Recall that Prob preserves weak pullbacks [58], thus its posetification Prob' can be described using the relation lifting as in Remark 4.8. In fact, for the probability functor, it happens that the relation lifting of a partial order is not just a preorder, but even a partial order [7]. Henceforth for a poset $X, \operatorname{Prob}^{\prime}(X)$ has the underlying set $\operatorname{Prob}\left(X_{0}\right)$, ordered as follows: for $p, p^{\prime} \in \operatorname{Prob}\left(X_{0}\right), p \leq p^{\prime}$ if and only if there is some $\omega \in \operatorname{Prob}\left(X_{0} \times X_{0}\right)$ such that $\sum_{x^{\prime} \in X} \omega\left(x, x^{\prime}\right)=p(x)$ and $\sum_{x \in X} \omega\left(x, x^{\prime}\right)=$ $p^{\prime}\left(x^{\prime}\right)$, and $\omega\left(x, x^{\prime}\right)>0 \Rightarrow x \leq x^{\prime}$.

\section{B. Characterising functors Pos $\rightarrow$ Pos in discrete arities.}

Recall from Proposition 3.4 that we have denoted, for each poset $X$, by $N_{X}$ the diagram

$$
D X_{1} \underset{D d^{0}}{\stackrel{D d^{1}}{\longrightarrow}} D X_{0}
$$

of discrete posets, where $X_{0}$ is the set of elements of $X, X_{1}$ is the set of all pairs $\left(x, x^{\prime}\right)$ with $x \leq x^{\prime}$ in $X$, while the maps $d^{0}, d^{1}$ are the obvious projections.

Theorem 4.12. For $T^{\prime}:$ Pos $\rightarrow$ Pos, the following are equivalent:

(1) There exists a functor $T:$ Set $\rightarrow$ Set such that $T^{\prime} \cong \operatorname{Lan}_{D}(D T)$, i.e., $T^{\prime}$ is a posetification of $T$.

(2) $T^{\prime}$ preserves discrete posets and coinserters of all diagrams $N_{X}$.

Proof. We prove first that the coinserters of diagrams $N_{X}$ form the density presentation of $D:$ Set $\rightarrow$ Pos in the sense of [33, Section 5.4]. Indeed, all coinserters of $N_{X}$ exist in Pos, the category Pos is the closure of Set under these coinserters, and the coinserters 
of $\left(N_{X}\right.$ are preserved by the functor $\operatorname{Pos}(D S,-):$ Pos $\rightarrow$ Pos. To see the latter, observe that for any set $S$, the poset $\operatorname{Pos}(D S, X)=X^{S}$ is a coinserter of

$$
\left(X_{1}\right)^{S} \underset{\left(d^{0}\right)^{S}}{\stackrel{\left(d^{1}\right)^{S}}{\longrightarrow}}\left(X_{0}\right)^{S}
$$

We prove now that (1) implies (2). Since $T D \cong D T^{\prime}$ holds, $T$ preserves discrete posets. By Equation (4.11), the collection of all coinserters of $\left(N_{X}\right.$ forms a density presentation of $D$, hence by [33, Theorem 5.29], $T$ preserves coinserters of all diagrams in $\left(N_{X}\right)$.

(2) implies (1). Since $T^{\prime}$ is assumed to preserve discrete posets, we may assume that $T^{\prime} D \cong$ $D T$ for some functor $T:$ Set $\rightarrow$ Set. Furthermore, by [33, Theorem 5.29], $T^{\prime} \cong \operatorname{Lan}_{D}\left(T^{\prime} D\right)$ holds. That is, $T^{\prime} \cong \operatorname{Lan}_{D}(D T)$ holds.

Recall that by Definition 4.7, a functor has a presentation in discrete arities if it is of the form $\operatorname{Lan}_{D} H$ for some $H$ : Set $\rightarrow$ Pos. Then we can drop in the theorem above the requirement that $T$ preserves discrete posets to obtain the following.

Theorem 4.13. A functor Pos $\rightarrow$ Pos has a presentation in discrete arities if and only if it preserves coinserters of all diagrams $\left(N_{X}\right)$.

Similarly, recalling Definition 3.9 of a strongly finitary functor, one has:

\section{Theorem 4.14.}

(1) A functor Pos $\rightarrow$ Pos is strongly finitary and preserves discrete posets if and only if it is the posetification of a finitary functor Set $\rightarrow$ Set.

(2) A functor Pos $\rightarrow$ Pos is strongly finitary if and only if it is a left Kan extension of a finitary functor Set $\rightarrow$ Pos.

Having in the above characterized functors Pos $\rightarrow$ Pos in discrete arities, we now turn to a special property that these have. First, recall that every endofunctor on Set preserves surjections [55] (assuming the axiom of choice). We shall establish below the correspondent result for Pos, recalling again Definition 4.7.

Proposition 4.15. Let $T^{\prime}:$ Pos $\rightarrow$ Pos have a presentation in discrete arities. Then $T^{\prime}$ preserves monotone surjections between posets.

Proof. Let $c: X \rightarrow Y$ be a surjective monotone map between posets. Then it is easy to see that $c$ is the (reflexive) coinserter of the comma object of $c$ with itself

$$
P \underset{d^{1}}{\stackrel{d^{0}}{\longrightarrow}} X \stackrel{c}{\longrightarrow} Y
$$

where $P$ is the poset of all pairs $\left(x, x^{\prime}\right)$ such that $c(x) \leq c\left(x^{\prime}\right)$, ordered component-wise, and $d^{0}, d^{1}$ are the canonical projections.

We want to show that $T^{\prime} c$ is again surjective. In fact, we shall see more: that $T^{\prime}$ preserves the coinserter (4.12). As $T^{\prime}$ is a left Kan extension along $D:$ Set $\rightarrow$ Pos, according to [33, Theorem 5.29], it is enough to check that (4.12) is $D$-absolute, that is, that (4.12) is preserved by $\operatorname{Pos}(D S,-)$ for every set $S$. To see the latter, let $S$ be an arbitrary set and form the diagram

$$
P^{S} \underset{\left(d^{1}\right)^{S}}{\stackrel{\left(d^{0}\right)^{S}}{\longrightarrow}} X^{S} \stackrel{c^{S}}{\longrightarrow} Y^{S}
$$


To prove that it is a coinserter, consider any monotone map $h: X^{S} \rightarrow Z$, with $h \circ\left(d^{0}\right)^{S} \leq$ $h \circ\left(d^{1}\right)^{S}$. Using the surjectivity of $c$, define $k: Y^{S} \rightarrow Z$ by $k\left(\left(y_{i}\right)_{i \in S}\right)=h\left(\left(x_{i}\right)_{i \in S}\right)$, where $c\left(\left(x_{i}\right)_{i \in S}\right)=\left(y_{i}\right)_{i \in S}$. Using the construction of the comma object $P$, one can easily check that the above does not depend on the choice of $\left(x_{i}\right)_{i \in S}$, and that the map $k$ thus defined is indeed monotone. Now the universal property of coinserters follows easily.

Example 4.16. We give an example of a Pos-functor which does not preserve surjections (and the definition of which involves a non-discrete arity). Let $T^{\prime}:$ Pos $\rightarrow$ Pos be the functor mapping a poset $X$ to the poset of monotone maps [2, $X]$ (equivalently, it could be written more intuitively as $X^{\mathbb{2}}$, the poset of ordered pairs in $X$, with the component-wise order). The fact that $[\mathcal{L}, X]$ only contains monotone maps has as a consequence that the surjection $f: D 2 \rightarrow \mathcal{2}$ mapping each of the 0,1 to itself, see below

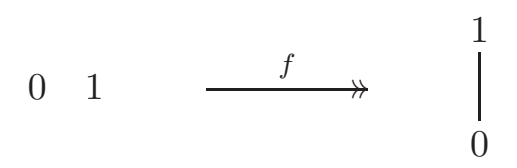

is not preserved by $T^{\prime}$. Indeed $T^{\prime} D 2$ has two elements, while $T^{\prime} \mathcal{T}$ has three elements.

\section{Presenting FunCtors on ordered varieties}

Coming back to the introduction, we remind the reader that our overall strategy is - starting with a functor $T:$ Set $\rightarrow$ Set for the type of coalgebras - to obtain from $T$ the Boolean logic $L:$ BA $\rightarrow$ BA by duality, and to obtain from the posetification $T^{\prime}$ of $T$, again by duality, the positive logic $L^{\prime}: \mathrm{DL} \rightarrow \mathrm{DL}$. The relationship between $L$ and $L^{\prime}$ will be studied in the next section. Here, we are going to make sure that the functors $L$ and $L^{\prime}$ obtained by abstract categorical constructions actually do have concrete presentations by operations and equations and thus correspond indeed to modal extensions of Boolean and positive propositional logic.

In the case of $L$, assuming that $L$ preserves sifted colimits, this is known already from [43] and we shall recall it below. In the case of $L^{\prime}$, we need to prove the enriched analogue of [43], which we shall obtain following the enriched generalization of [43] given in [57]. In particular, this enriched generalization will guarantee that $L^{\prime}$ can be presented by monotone operations. As a final twist, this enriched generalization would give us a presentation of $L^{\prime}$ using inequations over general ordered varieties. Therefore, it is important for us to show that, owing to the special nature of $\mathrm{DL}$, the enriched functor $L^{\prime}$ can equally be presented as the underlying ordinary functor $L_{o}^{\prime}$, which in turn has a presentation that does not rely on inequations.

5.A. Equational presentations of functors. We have seen, in Example 2.1, a presentation of a functor $L: \mathrm{BA} \rightarrow \mathrm{BA}$ and in Example 2.6 a presentation of a functor $L^{\prime}: \mathrm{DL} \rightarrow \mathrm{DL}$. Whereas it may be clear from these examples what we mean by a presentation, it is worth spending the effort to give a formal definition.

In what follows, $\mathscr{A}$ will denote a variety of algebras for a finitary signature. By a slight abuse of notation, we shall use the same notation as in case of the variety BA for the (monadic) adjunction

$$
F \dashv U: \mathscr{A} \rightarrow \text { Set }
$$


Denote by $\Sigma_{n}$ the set of $n$-ary modal operators, and by $\Gamma_{n}$ the set of equations in $n$ free variables. For instance, in Example 2.1 we have $\Sigma_{1}=\{\square\}$ and $\Sigma_{n}=\emptyset$ for $n \neq 1$, and $\Gamma_{0}=\{(\square \top, \top)\}, \Gamma_{2}=\{(\square(a \wedge b), \square a \wedge \square b)\}$ and $\Gamma_{m}=\emptyset$ for $m \neq 0,2$. Given a signature $\Sigma=\left(\Sigma_{n}\right)_{n<\omega}$, we write $\hat{\Sigma}$ : Set $\rightarrow$ Set for the corresponding polynomial functor

$$
X \mapsto \coprod_{n<\omega} \operatorname{Set}(n, X) \bullet \Sigma_{n}
$$

where $\bullet$ denotes the copower, see Section 3.C. Observe that with this notation, in Example 2.1 we have that $\Gamma_{n} \subseteq U F \hat{\Sigma} U F n \times U F \hat{\Sigma} U F n$ (interpret $U F X$ as the set of Boolean terms on $X$-generators).

Definition 5.1. [18, Definition 6] A functor $L$ on a variety $\mathscr{A}$ has a presentation by operations and equations, or, shortly, a presentation, if there are signatures $\Sigma$ and $\Gamma$, with $\Gamma_{n} \subseteq U F \hat{\Sigma} U F n \times U F \hat{\Sigma} U F n$, such that for all $A \in \mathcal{A}$ the following diagram, where $n$ ranges over natural numbers and $v$ ranges over all valuations $F n \rightarrow A$ (of $n$-variables in $A$ )

$$
F \hat{\Gamma} n \Longrightarrow F \hat{\Sigma} U F n \stackrel{F \hat{\Sigma} U v}{\longrightarrow} F \hat{\Sigma} U A \longrightarrow L A
$$

is a joint coequalizer.

Recall that a joint coequalizer of a family of parallel pairs with common codomain is an arrow which is a coequalizer of each pair of maps in that family.

The elements of the sets $\Gamma_{n}, n<\omega$, are often called the axioms, or equations of the presentation.

Remark 5.2 (Axioms of rank 1). We see that the format of the equations (i.e., the elements of $\Gamma_{n}$ ) requires them to be pairs in $U F \hat{\Sigma} U F n \times U F \hat{\Sigma} U F n$, that is, every variable must be under exactly one modal operator. Such equations are called equations, or axioms, of rank 1. For example, if we wanted to extend DL by negation (thinking of negation as a unary modal operator), then $\neg(a \wedge b)=\neg a \vee \neg b$ and $\neg 1=0$ are equations of rank 1 , but $a \wedge \neg a=0$ is not. The importance of equations of rank 1 is that they capture algebras for a functor (as opposed to general equations which correspond to algebras for a monad), see Theorem 5.4 below.

For proofs of the following proposition and theorem see [43, Theorem 4.7].

Proposition 5.3. A functor $L$ on a variety $\mathscr{A}$ has a presentation if and only if there are polynomial functors $\hat{\Sigma}, \hat{\Gamma}$ : Set $\rightarrow$ Set such that $L$ is a coequalizer

$$
F \hat{\Gamma} U \Longrightarrow F \hat{\Sigma} U \longrightarrow L
$$

in the category of endofunctors of $\mathscr{A}$.

Recall that any ordinary variety $\mathscr{A}$ can be presented by a signature $\Sigma_{\mathscr{A}}$ and equations $E_{\mathscr{A}}$. For instance the variety $\mathrm{DL}$ is presented by the constants $\perp, T$, the binary operations $\wedge, \vee$ and the usual equations defining distributive lattices, see e.g. [22].

Theorem 5.4. Let $L$ be an endofunctor of a variety $\mathscr{A}$. Let $\mathscr{A}$ be presented by a signature $\Sigma_{\mathscr{A}}$ and equations $E_{\mathscr{A}}$. Then:

(1) If $L$ has a presentation $\langle\Sigma, \Gamma\rangle$, then the category of L-algebras is isomorphic to the category of algebras for the signature $\Sigma_{\mathcal{A}}+\Sigma$ satisfying the equations $E_{\mathcal{A}}$ and $\Gamma$. 
(2) An endofunctor $L$ of a variety $\mathscr{A}$ has a presentation if and only if it preserves ordinary sifted colimits.

This theorem gives a bijection between endofunctors $L$ of a variety $\mathscr{A}$ that preserve sifted colimits and logics extending $\mathscr{A}$ by 'modal operators' and axioms of rank 1 . The theorem enables us to investigate such logics using purely category theoretic means.

5.B. Equational presentations of locally monotone functors. For the purposes of our investigations, we are interested in modal logics extending DL, given by rank 1 axioms of monotone operations. While $U: \mathrm{DL} \rightarrow$ Set is certainly finitary and monadic (since DL is an ordinary variety of algebras), it is also the case that the natural forgetful functor $U^{\prime}: \mathrm{DL} \rightarrow$ Pos, mapping a distributive lattice to its carrier equipped with the lattice order, exhibits DL as an ordered variety.

For now, let us be slightly more general and consider an ordered variety

$$
F^{\prime} \dashv U^{\prime}: \mathscr{A} \rightarrow \text { Pos }
$$

By an ordered signature $\Sigma^{\prime}$ we shall mean a family of posets $\Sigma^{\prime}=\left(\Sigma_{n}^{\prime}\right)_{n<\omega}$. Denote by $\tilde{\Sigma}^{\prime}:$ Pos $\rightarrow$ Pos the corresponding polynomial functor

$$
X \mapsto \coprod_{n<\omega} \operatorname{Pos}(D n, X) \bullet \Sigma_{n}^{\prime}
$$

Recall that - denotes the copower, which in the case above is just the cartesian tensor product in Pos.

In the following, we shall call a functor Pos $\rightarrow$ Pos polynomial only if it is of the form $\tilde{\Sigma}^{\prime}$, for some ordered signature $\Sigma^{\prime}$. Notice that a polynomial functor only employs discrete arities, and that if $\Sigma^{\prime}=D \Sigma$ for some (necessarily unique!) Set-signature $\Sigma$, then $\tilde{\Sigma}^{\prime}$ is the posetification of $\hat{\Sigma}$ in the sense of Definition 4.1.

Definition 5.5. A functor $L^{\prime}$ on an ordered variety $\mathscr{A}$ has an ordered presentation in discrete arities, or, shortly, an ordered presentation, if there are ordered signatures $\Sigma^{\prime}$ and $\Gamma^{\prime}$, with $\Gamma_{n}^{\prime} \subseteq U^{\prime} F^{\prime} \tilde{\Sigma}^{\prime} U^{\prime} F^{\prime} D n \times U^{\prime} F^{\prime} \tilde{\Sigma}^{\prime} U^{\prime} F^{\prime} D n$ for each natural number $n$, such that for all $A$ in $\mathscr{A}$, the following diagram, where $n$ ranges again over natural numbers and $v$ ranges over all valuations $F^{\prime} D n \rightarrow A$ (of $n$-variables in $A$ )

$$
F^{\prime} \tilde{\Gamma}^{\prime} D n \Longrightarrow F^{\prime} \tilde{\Sigma}^{\prime} U^{\prime} F^{\prime} D n \stackrel{F^{\prime} \tilde{\Sigma}^{\prime} U^{\prime} v}{\longrightarrow} F^{\prime} \tilde{\Sigma}^{\prime} U^{\prime} A \stackrel{q}{\longrightarrow} L^{\prime} A
$$

is a joint coequalizer.

In the definition above it is not important to allow $\Gamma_{n}^{\prime}$ to be posets. On other hand, for a general variety $\mathscr{A}$ it is important to allow the $\Sigma_{n}^{\prime}$ to be posets (which is the reason why we can use a coequalizer in (5.2) instead of a coinserter). Then again, for $\mathscr{A}=\mathrm{DL}$ we can take the $\Sigma_{n}^{\prime}$ discrete since for DL the order is equationally definable, as will be discussed in detail in Section 5.D.

Remark 5.6. An ordered presentation is monotone. In detail, let $\alpha: L^{\prime} A \rightarrow A$ be an algebra. Consider an operation $\sigma \in \Sigma_{n}^{\prime}$ and $a, a^{\prime}: D n \rightarrow U^{\prime} A$ with $a \leq a^{\prime}$, that is, $a_{i} \leq a_{i}^{\prime}$ for all $1 \leq i \leq n$. We have to show that $\sigma(a) \leq \sigma\left(a^{\prime}\right)$ holds in the $L^{\prime}$-algebra $(A, \alpha)$. But this is equivalent to the obvious inequality $U^{\prime} \alpha \circ q^{b}(\sigma, a) \leq U^{\prime} \alpha \circ q^{b}\left(\sigma, a^{\prime}\right)$ where $q^{b}: \tilde{\Sigma}^{\prime} U^{\prime} A \rightarrow U^{\prime} L^{\prime} A$ is the adjoint transpose of the quotient map $q: F^{\prime} \tilde{\Sigma}^{\prime} U^{\prime} A \rightarrow L^{\prime} A$ and $(\sigma, a)$ and $\left(\sigma, a^{\prime}\right)$ are pairs in $\Sigma_{n}^{\prime} \bullet \operatorname{Pos}\left(D n, U^{\prime} A\right)=\Sigma_{n}^{\prime} \times \operatorname{Pos}\left(D n, U^{\prime} A\right)$. 
Proposition 5.7. A locally monotone functor $L^{\prime}$ on an ordered variety $\mathscr{A}$ has an ordered presentation if and only if there are polynomial functors $\tilde{\Sigma}, \tilde{\Gamma}:$ Pos $\rightarrow$ Pos such that $L$ is a coequalizer

$$
F^{\prime} \tilde{\Gamma} U^{\prime} \longrightarrow F^{\prime} \tilde{\Sigma} U^{\prime} \longrightarrow L^{\prime}
$$

in the category of locally monotone endofunctors.

Proof. See [57, Theorem 3.18] where the more general situation was considered.

Theorem 5.8. Let $L^{\prime}: \mathscr{A} \rightarrow \mathscr{A}$ be a locally monotone functor on an ordered variety. Then $L^{\prime}$ preserves Pos-sifted colimits if and only if it has an ordered presentation in discrete arities.

Proof. The proof is essentially contained in [57], but for the reader's convenience, we shall spell out some of the details.

We denote by $\mathscr{A}_{f f}$ the full subcategory of $\mathscr{A}$ spanned by the algebras which are free on finite discrete posets and by $F_{f}^{\prime}: \operatorname{Set}_{f} \rightarrow \mathscr{A}_{f f}$ the corresponding domain-codomain restriction of the composite $F^{\prime} D$ : Set $\rightarrow$ Pos $\rightarrow \mathscr{A}$. Then we follow the next steps:

(1) Observe first that $\left[F_{f}^{\prime}, U^{\prime}\right]:\left[\mathscr{A}_{f f}, \mathscr{A}\right] \rightarrow\left[\operatorname{Set}_{f}\right.$, Pos $]$, sending a functor $L^{\prime}: \mathscr{A}_{f f} \rightarrow \mathscr{A}$ to the composite $U^{\prime} L^{\prime} F_{f}^{\prime}$, is of descent type. That is, $\left[F_{f}^{\prime}, U^{\prime}\right]$ is right adjoint and each component of the counit of the corresponding adjunction is a coequalizer [57, Lemma 3.14].

(2) The functor $[E,-]:\left[\operatorname{Set}_{f}, \mathrm{Pos}\right] \rightarrow\left[\left|\operatorname{Set}_{f}\right|, \operatorname{Pos}\right]$, where $\left|\operatorname{Set}_{f}\right|$ is the discrete category of finite sets and $E:\left|\operatorname{Set}_{f}\right| \rightarrow$ Set $_{f}$ is the inclusion, is monadic (again, this follows from Lemma 3.14 of [57]).

(3) Consequently, the composite

$$
\left[\mathscr{A}_{f f}, \mathscr{A}\right] \stackrel{\left[F_{f}^{\prime}, U^{\prime}\right]}{\longrightarrow}\left[\operatorname{Set}_{f}, \text { Pos }\right] \stackrel{[E,-]}{\longrightarrow}\left[\mid \text { Set }_{f} \mid, \text { Pos }\right]
$$

is of descent type [57, Theorem 3.18].

Therefore, every locally monotone functor $L^{\prime}: \mathscr{A}_{f f} \rightarrow \mathscr{A}$ (i.e., every $L^{\prime}$ preserving sifted colimits) arises as a coequalizer as in Proposition 5.7, that is, it admits an ordered presentation in discrete arities.

5.C. Ordinary and ordered presentations of functors on BA. Let $\mathscr{A}$ be a Posenriched category with discretely ordered hom-posets, such as BA. Then, as we are going to show now, there is no essential difference in the presentations according to Sections $5 . B$ and 5.A.

Before coming to functors on varieties, let us clarify when ordinary varieties are ordered varieties with discrete hom-sets and vice versa. Recall that we wrote $C \dashv D$ : Set $\rightarrow$ Pos for the adjunction in which $D$ is the discrete functor and $C$ the connected components functor.

Proposition 5.9. Let $F^{\prime} \dashv U^{\prime}: \mathscr{A} \rightarrow$ Pos be an ordered variety. It has discretely ordered hom-posets if and only if any of the following equivalent conditions are satisfied.

(1) $U^{\prime}$ factors through $D$ : Set $\rightarrow$ Pos.

(2) $U^{\prime} \cong D C U^{\prime}$.

(3) $\eta_{U^{\prime}}: U^{\prime} \rightarrow D C U^{\prime}$ is an isomorphism, where $\eta$ is the unit of the adjunction $C \dashv D$. 
If any of the above conditions is satisfied then also $F^{\prime}$ factors through $C$ : Pos $\rightarrow$ Set via $F^{\prime} \cong F^{\prime} D C$. Moreover, $F^{\prime} D \dashv C U^{\prime}: \mathscr{A} \rightarrow$ Set is monadic.

Conversely, if $F \dashv U: \mathscr{A} \rightarrow$ Set is an ordinary variety and the only order on algebras in $\mathscr{A}$ making all operations monotone is the trivial discrete order (as it is the case in BA), then $F C \dashv D U: \mathscr{A} \rightarrow$ Pos is an ordered variety, see [45].

Proposition 5.10. Let $\mathscr{A} \rightarrow$ Pos be a variety with discretely ordered homsets and let $L: \mathscr{A} \rightarrow \mathscr{A}$ be a (necessarily locally monotone) functor. Then the functor $L$ preserves ordinary sifted colimits if and only if $L$ preserves Pos-enriched sifted colimits. Moreover, $\langle D \Sigma, D \Gamma\rangle$ is an ordered presentation of $L$ if and only if $\langle\Sigma, \Gamma\rangle$ is a presentation of $L$ and $\left\langle\Sigma^{\prime}, \Gamma^{\prime}\right\rangle$ is an ordered presentation of $L$ if and only if $\left\langle C \Sigma^{\prime}, C \Gamma^{\prime}\right\rangle$ is a presentation of $L$.

The proposition above guarantees that for a functor $L: \mathrm{BA} \rightarrow \mathrm{BA}$, it does not matter whether we consider it as an ordinary functor on the variety BA, or whether we consider it as a locally monotone functor on the ordered variety BA.

5.D. Ordinary and ordered presentations of functors on DL. The aim of this section is to show that not only does an endofunctor of DL have a presentation by operations and equations if and only if it preserves ordinary sifted colimits, but also that a functor has a presentation by monotone operations and equations if and only if it preserves enriched sifted colimits.

We begin with the following:

Proposition 5.11. If $\mathscr{A}$ is an ordered variety and $L^{\prime}: \mathscr{A} \rightarrow \mathscr{A}$ is a locally monotone functor which preserves enriched sifted colimits, then the underlying ordinary functor $L_{o}^{\prime}$ : $\mathscr{A}_{0} \rightarrow \mathscr{A}_{0}$ preserves ordinary sifted colimits.

Proof. By [45, Theorem 6.9] we know that $\mathscr{A}$ is a free cocompletion by enriched sifted colimits of the full subcategory $\mathbf{J}^{\prime}: \mathscr{A}_{f f} \hookrightarrow \mathscr{A}$ spanned by free algebras on finite and discrete sets of generators.

Furthermore, $\mathbf{J}^{\prime}: \mathscr{A}_{f f} \hookrightarrow \mathscr{A}$ has the density presentation consisting of the three classes below:

(1) reflexive coinserters,

(2) (conical) filtered colimits, i.e., by colimits weighted by $\varphi: \mathscr{D}^{\circ p} \rightarrow$ Pos where $\mathscr{D}$ is ordinary filtered category and $\varphi$ is the constant functor at the one-element poset,

(3) reflexive coequalizers.

The reason is that we can

(1) use coinserters of truncated nerves to create algebras, free on any finite poset,

(2) use (conical) filtered colimits to obtain free algebras on any poset,

(3) use a reflexive coequalizer (that is, a canonical presentation) to obtain any algebra.

Hence, we know that $L^{\prime}: \mathscr{A} \rightarrow \mathscr{A}$ preserves enriched sifted colimits if and only if $L^{\prime}$ preserves colimits in (1), (2) and (3). Since colimits in (2) and (3) are conical, they are preserved by $L_{o}^{\prime}$. But $\mathscr{A}$ is Pos-cocomplete, being an ordered variety, hence $\mathscr{A}_{0}$ is Set-cocomplete, according to [33, Section 3.8]. And a functor between ordinary cocomplete categories preserves sifted colimits if and only if it preserves ordinary filtered colimits and reflexive coequalizers [5, Theorem 7.7]. 
The above proposition, together with Theorem 5.4 imply that in case $\mathscr{A}_{0}$ is an ordinary variety, the underlying ordinary functor $L_{o}^{\prime}$ also has a presentation by operations and equations.

Example 5.12. Let $L^{\prime}: \mathrm{DL} \rightarrow \mathrm{DL}$ be the locally monotone functor presented by one unary operation, written as $\square$, and no equations. It follows from the proposition that monotonicity of $\square$ is equationally definable. Explicitly, the induced equational presentation of $L_{o}^{\prime}$ can be given by

$$
\square a \wedge \square(a \vee b)=\square a
$$

Of course, the proposition only tells us that all finitary equations valid for a monotone together present $L_{o}^{\prime}$. But it is not difficult to check that the equation above is enough to force $\square$ to be monotone.

Conversely, if $\mathscr{A}_{O}$ is an ordinary variety, it makes sense to ask how a presentation of a functor on $\mathscr{A}_{0}$ induces a presentation of a functor on $\mathscr{A}$. We are thinking of a situation such as the one of Example 2.6. where the functor $L^{\prime}$ is defined equationally on the ordinary variety $\mathrm{DL}_{o}$, but can also be seen as a locally monotone functor on the ordered variety DL. Consider thus an ordered variety $F^{\prime} \dashv U^{\prime}: \mathscr{A} \rightarrow$ Pos. By slight abuse of notation, we let $\mathscr{A}_{0}$ to stand both for the underlying ordinary category of $\mathscr{A}$, and for the Pos-subcategory which has the same objects and arrows as $\mathscr{A}$ but discrete homsets, and we put $\tilde{D}: \mathscr{A}_{O} \rightarrow \mathscr{A}$ to be the (necessarily locally monotone) inclusion. Assume that $\mathscr{A}_{O}$ is an ordinary variety. Then to say that the following diagram

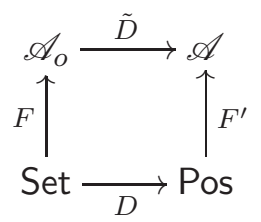

commutes defines $F$. Let $\langle\Sigma, \Gamma\rangle$ be a presentation of a functor $L_{o}: \mathscr{A}_{O} \rightarrow \mathscr{A}_{O}$ by the coequalizer (5.1) of Definition 5.1. It induces a presentation $\left\langle\Sigma^{\prime}=D \Sigma, \Gamma^{\prime}=D \Gamma\right\rangle$ of a functor $L^{\prime}: \mathscr{A} \rightarrow \mathscr{A}$ by the coequalizer (5.2) of Definition [5.5. Since $\tilde{D}$ preserves the coequalizer (5.1), we obtain for all $A \in \mathscr{A}$ the dotted arrow in the diagram

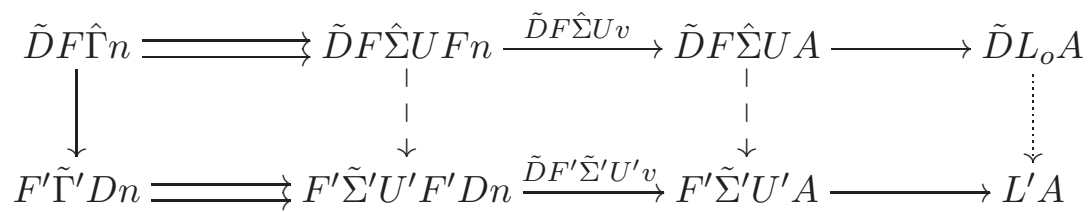

Due to $\tilde{D} F=F^{\prime} D$ and $D \hat{\Gamma}=\tilde{\Gamma}^{\prime} D$, the left-most vertical arrow is an isomorphism. Even though the two vertical dashed arrows in the middle need not be order-reflecting, they are surjective, which implies that also the the dotted vertical arrow on the right is onto. But since the lower row may have more inequalities, the dotted arrow need not be bijective, see Example 5.14 below.

Definition 5.13. We say that $\langle\Sigma, \Gamma\rangle$ is a presentation by monotone operations and equations, or, shortly, a monotone presentation, if the dotted arrow (5.3) is an isomorphism for all $A \in \mathscr{A}$. 
This terminology is justified by Remark 5.6, according to which $\langle D \Sigma, D \Gamma\rangle$ is a presentation by monotone operations.

Example 5.14. The presentation of Example 2.6 is a presentation by monotone operations, since to say that $\square$ preserves meets and that $\diamond$ preserves joins forces $\square$ and $\diamond$ to be monotone. On the other hand, if these axioms had been omitted from the presentation, the resulting presentation would not have been monotone.

To summarize, given an ordered presentation $\left\langle\Sigma^{\prime}, \Gamma^{\prime}\right\rangle$ of a functor $L^{\prime}: \mathscr{A} \rightarrow \mathscr{A}$ on an ordered variety in the sense of Definition 5.5, there is a monotone presentation by operations and equation $\langle\Sigma, \Gamma\rangle$ of the underlying ordinary functor $L_{o}^{\prime}$ if $\mathscr{A}_{O} \rightarrow \operatorname{Pos}_{o} \rightarrow$ Set is a variety. This is due to the following result.

Theorem 5.15. Let $L$ be an endofunctor on a category $\mathscr{A}$ that is both an ordered and an ordinary variety. If $L$ has an ordered presentation, then it has a presentation by monotone operations and equations.

Proof. To say that $\mathscr{A}$ is both an ordered and an ordinary variety is to say that $\mathscr{A}$ comes equipped with a forgetful functor $\mathscr{A} \rightarrow$ Pos so that $\mathscr{A} \rightarrow$ Pos is an ordered variety and $\mathscr{A}_{O} \rightarrow$ $\mathrm{Pos}_{O} \rightarrow$ Set is an ordinary variety. If $L$ has an ordered presentation then it preserves enriched sifted colimits, hence $L_{o}$ preserves ordinary sifted colimits, hence $L_{o}$ has a presentation.

We can now conclude what we shall need to know about functors on DL.

Theorem 5.16. For a locally monotone functor $L^{\prime}: \mathrm{DL} \rightarrow \mathrm{DL}$ the following are equivalent:

(1) $L^{\prime}$ has a presentation by monotone operations and equations.

(2) $L^{\prime}$ preserves Pos-enriched sifted colimits.

(3) $L^{\prime}$ is the Pos-enriched left Kan extension of its restriction to discretely finitely generated free distributive lattices.

As in Proposition 2.4, we now obtain that

Corollary 5.17. If $T^{\prime}$ is the the convex powerset functor, then the functor $L^{\prime}$ of Example[2.6 is isomorphic to the sifted colimits preserving functor $\mathbf{L}^{\prime}$ whose restriction to $\mathrm{DL}_{f f}$ is $P^{\prime} T^{\prime \mathrm{op}} S^{\prime}$ as in Equation (2.5).

\section{Positive CoAlgebraic logic}

The reader might find it useful to consult Section 6.C first, even though it relies on some notation introduced in the next two subsections. 
6.A. Morphisms of logical connections. We recall the logical connections (dual adjunctions, see [44) mentioned in Section 2 between sets and Boolean algebras, and between posets and distributive lattices. Both are considered to be Pos-enriched, where for the first logical connection the enrichment is discrete. They are related as follows:

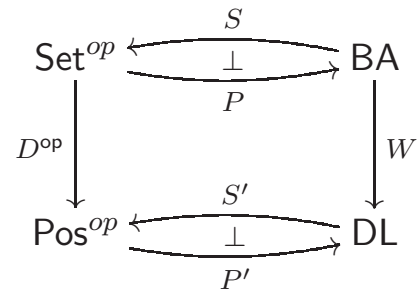

In the top row of the above diagram, we recall again for the reader's convenience that $P$ is the contravariant powerset functor, while $S$ maps a Boolean algebra to its set of ultrafilters. The bottom row has $P^{\prime}$ mapping a poset to the distributive lattice of its upsets, and $S^{\prime}$ associating to each distributive lattice the poset of its prime filters. As for the pair of functors connecting the two logical connections: $D$ was introduced earlier as the discrete functor, while $W$ is the functor associating to each Boolean algebra its underlying distributive lattice.

It is easy to see that the pair $\left(D^{\mathrm{op}}, W\right)$ is a morphism of adjunctions in the sense of 47 , $\S$ IV.7]. This means that the following diagrams commute, and that the coherence condition below holds:
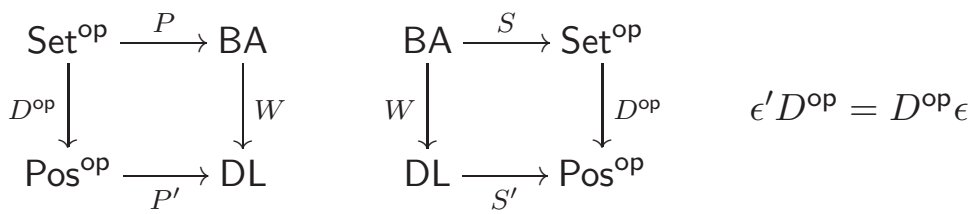

where $\epsilon$ and $\epsilon^{\prime}$ are the counits of $S \dashv P$ and $S^{\prime} \dashv P^{\prime}$, respectively.

Remark 6.1. It will turn out useful later to use not only that $D$ has, as mentioned in Section 3.B. as a Pos-enriched left adjoint the connected components functor $C$, but also that $W$ has a Pos-enriched right adjoint $K$, mapping a distributive lattice $A$ to the Boolean algebra of complemented elements in $A$, also known as the centre of $A$, see [14]. Then the mate of the first square in (6.2) under the above adjunctions, namely $P C^{\mathrm{op}} \rightarrow K P^{\prime}$, is in fact an isomorphism (to see this, use that the connected components of a poset are precisely its minimal subsets which are both upward and downward closed).

6.B. Positive coalgebraic logic. We shall now expand the propositional logics BA and DL by modal operators. We start with an endofunctor $T$ of Set in the top left-hand corner of (6.1), and an endofunctor $T^{\prime}$ of Pos in the bottom left-hand corner. We are mostly interested in the case where $T^{\prime}:$ Pos $\rightarrow$ Pos is the posetification of $T$ (see Definition 4.1) and $L: \mathrm{BA} \rightarrow \mathrm{BA}$ and $L^{\prime}: \mathrm{DL} \rightarrow \mathrm{DL}$ are (the functors of) the associated logics as in (2.3) and (2.6), in which case we denote the logics by boldface letters $\mathbf{L}$ and $\mathbf{L}^{\prime}$.

But some of the following hold under the weaker assumptions that $T^{\prime}$ is an arbitrary extension of $T$, and that $L$ and $L^{\prime}$ are arbitrary coalgebraic logics for $T$ and $T^{\prime}$, respectively. 
Let therefore $T$ be an endofunctor of Set, and $T^{\prime}$ be an extension of $T$ to Pos as in (4.1). Logics for $T$, respectively $T^{\prime}$ are given by functors $L: \mathrm{BA} \rightarrow \mathrm{BA}$ and $L^{\prime}: \mathrm{DL} \rightarrow \mathrm{DL}$ and by natural transformations

$$
\delta: L P \rightarrow P T^{\mathrm{op}} \quad \delta^{\prime}: L^{\prime} P^{\prime} \rightarrow P^{\prime} T^{\prime \mathrm{op}}
$$

Intuitively, $\delta$ and $\delta^{\prime}$ assign to the syntax given by (presentations of) $L$ and $L^{\prime}$ the corresponding one-step semantics in subsets, respectively upsets. To compare $L$ and $L^{\prime}$ we need the isomorphism $\alpha: D T \rightarrow T^{\prime} D$ from (4.1) saying that $T^{\prime}$ extends $T$, and also the relation $W P=P^{\prime} D^{\mathrm{op}}$ from (6.2) (which formalizes the trivial observation that taking all upsets of a discrete set is the same as taking all subsets). Referring back to the introduction, we now make the following definition.

Definition 6.2. We say that a logic $\left(L^{\prime}, \delta^{\prime}\right)$ for $T^{\prime}$ is a positive fragment of the logic $(L, \delta)$ for $T$, if there is a natural transformation $\beta: L^{\prime} W \rightarrow W L$ with $W \delta \circ \beta P=P^{\prime} \alpha^{\mathrm{op}} \circ \delta^{\prime} D^{\mathrm{op}}$, or, in diagrams

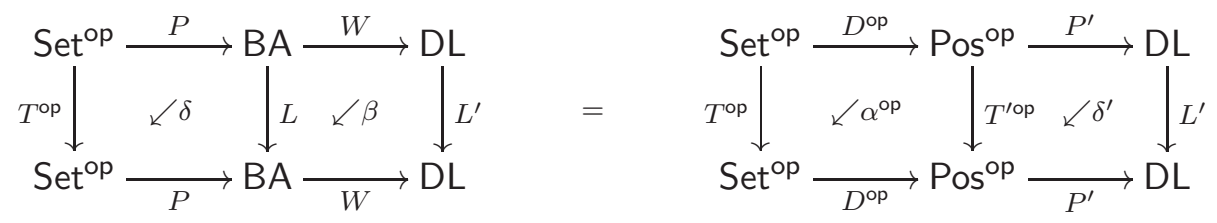

We call $\left(L^{\prime}, \delta^{\prime}\right)$ the maximal positive fragment of $(L, \delta)$ if $\beta$ is an isomorphism.

Recall that we have defined in Sections 2.G and 2.H, respectively, the logics $\mathbf{L}, \mathbf{L}^{\prime}$ induced by $T$ and (an extension) $T^{\prime}$ as $\mathbf{L}=P T^{\mathrm{op}} S$ and $\mathbf{L}^{\prime}=P^{\prime} T^{\prime \mathrm{op}} S^{\prime}$ on discretely finitely generated free objects. As explained in the introduction, our desired result is to prove that a certain canonically given $\mathbf{L}^{\prime} W \rightarrow W \mathbf{L}$, denoted by $\boldsymbol{\beta}$, is an isomorphism. The difficulty, as well as the need for the proviso that $T$ preserves weak pullbacks, stems from the fact that in DL (as opposed to BA) the class of functors determined on free algebras on finitely many discrete generators is strictly smaller than the class of functors determined on finitely presentable (=finite) algebras, as Example 6.6 will show.

As stepping stones, therefore, we first investigate what happens in the cases where

- the functor $L^{\prime}$ is determined by $P^{\prime} T^{\prime \text { op }} S^{\prime}$ on all algebras,

- the functor $\bar{L}^{\prime}$ is determined by $P^{\prime} T^{\text {op }} S^{\prime}$ on the full subcategory $\mathrm{DL}_{f}$ of finitely presentable algebras,

before turning to the situation of the functor $\mathbf{L}^{\prime}$ determined by $P^{\prime} T^{\prime \text { op }} S^{\prime}$ on the full subcategory $\mathrm{DL}_{f f}$ of strongly finitely presentable algebras (=free algebras on finitely many discrete generators).

6.C. Overview. To summarize the situation, consider

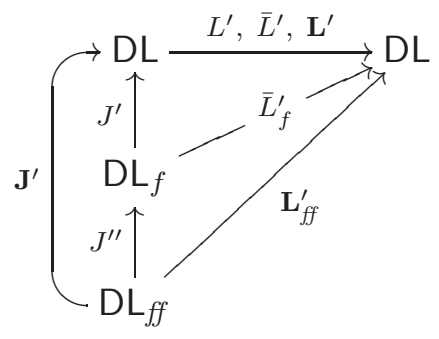


where we use subscripts to show the restrictions of $\bar{L}^{\prime}$ and $\mathbf{L}^{\prime}$ to the corresponding subcategories of DL where these functors coincide by definition with $P^{\prime} T^{\prime \text { op }} S^{\prime}$.

Section 6.D treats the case of $L^{\prime}$. Using the dual adjunction of DL and Pos, one easily obtains the required isomorphism $\beta: L^{\prime} W \rightarrow W L$. But on the other hand, presenting the logic of $L^{\prime}$ would require operations of infinite arity.

Section 6.E achieves the restriction to finitary syntax, by reducing $L^{\prime}$ to a functor $\bar{L}^{\prime}: \mathrm{DL} \rightarrow \mathrm{DL}$ that is determined by the action $P^{\prime} T^{\prime \mathrm{op}} S^{\prime}$ on the full subcategory $\mathrm{DL}_{f}$ on finitely presentable distributive lattices. This setting has the advantage that $\mathrm{DL}_{f}$ is dually equivalent to $\operatorname{Pos}_{f}$, from which we obtain again the required isomorphism $\bar{\beta}: \bar{L}^{\prime} W \rightarrow W \bar{L}$. On the other hand, such functors $\bar{L}^{\prime}$ are in general not presentable by monotone operations and equations.

Finally, Section 6.F restricts to the category $\mathrm{DL}_{f f}$ of discretely finitely generated free distributive lattices, which guarantees that the logic of $\mathbf{L}^{\prime}$ can be indeed presented by monotone operations and equations, and that the category of $\mathbf{L}^{\prime}$-algebras is a(n ordered) variety of modal algebras. In order to obtain that the corresponding $\boldsymbol{\beta}: \mathbf{L}^{\prime} W \rightarrow W \mathbf{L}$ is an isomorphism, we give conditions under which the functors $\bar{L}^{\prime}$ and $\mathbf{L}^{\prime}$ coincide. This is done by determining when $\bar{L}_{f}^{\prime}$ in (6.4) is a left Kan extension of its restriction along $J^{\prime \prime}: \mathrm{DL}_{f f} \rightarrow \mathrm{DL}_{f}$.

6.D. The case of $L^{\prime}=P^{\prime} T^{\text {op }} S^{\prime}$ on all algebras. We shall associate to any extension $\alpha: D T \rightarrow T^{\prime} D$ the pairs $(L, \delta)$ and $\left(L^{\prime}, \delta^{\prime}\right)$ corresponding to $T$ and $T^{\prime}$ respectively, with $L=P T^{\mathrm{op}} S$ and $\delta=P T^{\mathrm{op}} \epsilon: P T^{\mathrm{op}} S P \rightarrow P T^{\mathrm{op}}, L^{\prime}=P^{\prime} T^{\prime \mathrm{op}^{\prime}} S^{\prime}$ and $\delta^{\prime}$ being defined analogously. We then immediately obtain an isomorphism $\beta$ by the following:

Proposition 6.3. Given an extension $\alpha: D T \rightarrow T^{\prime} D$, the natural isomorphism $\beta: L^{\prime} W \rightarrow$ $W L$ given by the composite below

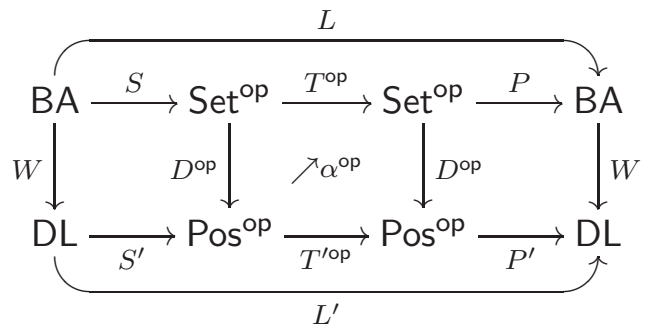

exhibits $L^{\prime}=P^{\prime} T^{\prime \mathrm{op}} S^{\prime}$ as the maximal positive fragment of $L=P T^{\mathrm{op}} S$.

Proof. This follows from ( $D^{\mathrm{op}}, W$ ) being a morphism of adjunctions (see (6.2) ).

6.E. The case of $\bar{L}^{\prime}=P^{\prime} T^{\prime \mathrm{op}} S^{\prime}$ on finitely presentable algebras. A similar result holds if we define logics via $P T^{\mathrm{op}} S A$ for finitely presentable $A$, as we are going to show now. To this end, we use the subscript $(-)_{f}$ to denote the restriction of categories and (domaincodomain) functors to finite objects as e.g. when writing as earlier the dense inclusions $I:$ Set $_{f} \rightarrow$ Set, $I^{\prime}: \operatorname{Pos}_{f} \rightarrow$ Pos, $J: \mathrm{BA}_{f} \rightarrow \mathrm{BA}$ and $J^{\prime}: \mathrm{DL}_{f} \rightarrow \mathrm{DL}$.

Since Pos is locally finitely presentable as a closed category, and the underlying ordinary categories $\mathrm{Set}_{o}, \mathrm{DL}_{o}, \mathrm{BA}_{o}$ are also locally finitely presentable, it follows from [34 that the 
finitely presentable objects in all the above categories are precisely the same as in the ordinary case i.e., the ones for which the underlying sets are finite.

Note that we have the following commuting diagram

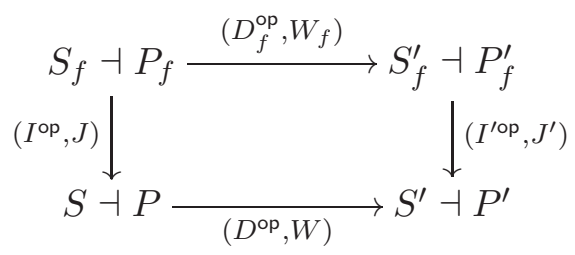

in the category of transformations of adjoints.

Define now $(\bar{L}, \bar{\delta})$ for $T$ as

$$
\bar{L}=\operatorname{Lan}_{J}\left(P T^{\mathrm{op}} S J\right) \quad \text { and } \quad \bar{\delta}: \bar{L} P \rightarrow P T^{o p}
$$

as the adjoint transpose of $\bar{L} \rightarrow P T^{\mathrm{op}} S$ arising from the universal property of the left Kan extension $\bar{L}$. By construction, $\bar{L}$ is finitary and is given by $P T^{\mathrm{op}} S$ on finite(ly presentable) Boolean algebras. Similarly, obtain $\left(\bar{L}^{\prime}, \bar{\delta}^{\prime}\right)$ for $T^{\prime}$ on distributive lattices.

The forgetful functor $W: \mathrm{BA} \rightarrow \mathrm{DL}$ is finitary, being a left adjoint (Section 6.A). Thus in order to obtain an (iso)morphism $\bar{\beta}: \bar{L}^{\prime} W \rightarrow W \bar{L}$ between finitary functors, it will be enough to provide its restriction along $J$ to finitely presentable objects. But we can get such a transformation from the isomorphism of Proposition 6.3, namely

$$
\bar{\beta}_{f}: \bar{L}^{\prime} W J \cong \bar{L}^{\prime} J^{\prime} W_{f} \cong P^{\prime} T^{\prime \mathrm{op}} S^{\prime} J^{\prime} W_{f} \cong W P T^{\mathrm{op}} S J \cong W \bar{L} J
$$

where the second and the last isomorphisms are given by the units of left Kan extensions.

Remark 6.4. Recall the definition of $\mathbf{L}$ from Equation (2.3). Since every finitely presentable non-trivial Boolean algebra is a retract of a finitely generated free algebra, we can identify $\mathbf{L}=\bar{L}$, see e.g. [43, Proposition 3.4].

According to the above, we then have

Proposition 6.5. The isomorphism $\bar{\beta}$ exhibits $\bar{L}=\operatorname{Lan}_{J^{\prime}}\left(P^{\prime} T^{\prime \mathrm{op}} S^{\prime} J^{\prime}\right)$ as the maximal positive fragment of $(\mathbf{L}, \delta)$.

Proof. The easiest way to check Equation (6.3) is to show that $\bar{\beta}_{f}$, defined by Equation (6.6), fulfills
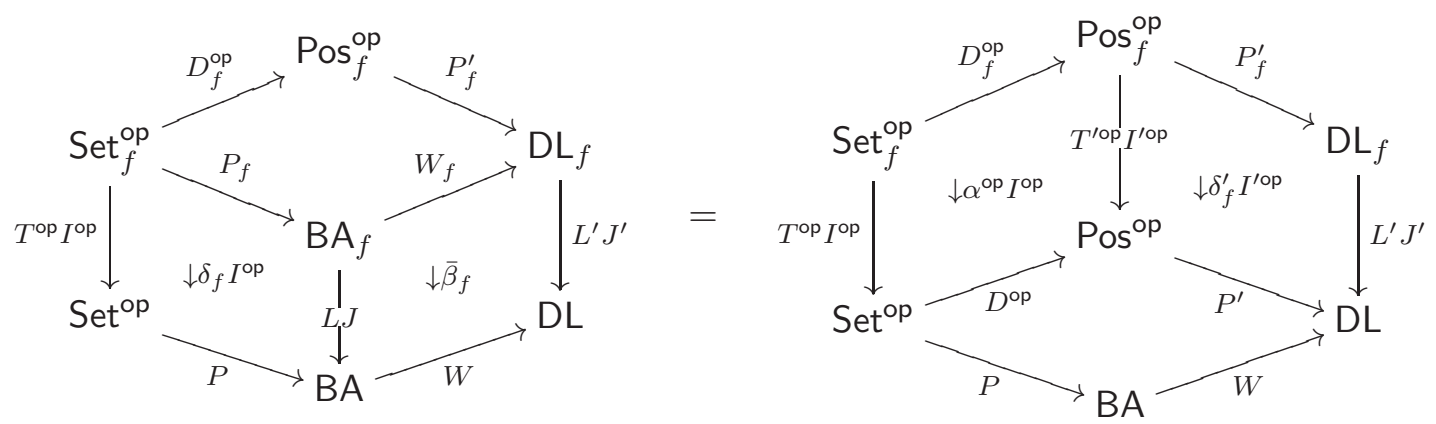

But this follows from Proposition 6.3 and Equation (6.5). 
This proposition does not yet give us the desired result, as the endofunctor $\bar{L}^{\prime}$ on $\mathrm{DL}$ is not necessarily determined by its action on discretely finitely generated free algebras and, therefore, is not guaranteed to be equationally definable and to give rise to a variety of modal algebras.

Example 6.6. We give an example of a locally monotone functor $\bar{L}^{\prime}: D L \rightarrow D L$ that is finitary but does not preserve sifted colimits i.e., it is not determined by its action on discretely finitely generated free algebras. Consider the composite (comonad)

$$
\bar{L}^{\prime}: \mathrm{DL} \stackrel{K}{\longrightarrow} \mathrm{BA} \stackrel{W}{\longrightarrow} \mathrm{DL}
$$

where $K$ is the right adjoint of the forgetful functor $W$ (recall that we met $K$ in Remark6.1 assigning to each distributive lattice $A$ the Boolean subalgebra $K A$ of its complemented elements). We shall exhibit below a reflexive coinserter that it is not preserved by $\bar{L}^{\prime}$. Since reflexive coinserters are sifted colimits, this means that $\bar{L}^{\prime}: \mathrm{DL} \rightarrow \mathrm{DL}$ does not preserve (all) sifted colimits.

Let $e: D 2 \rightarrow X$ be the obvious embedding of the two-element discrete poset into the poset $X$ with three elements 0,1 and $T$, satisfying $0<\top$ and $1<\top$. Then $e$ is a coreflexive inserter in Pos of the pair $f, g: X \rightarrow Y$, where $Y$ is the poset

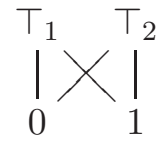

and $f(0)=g(0)=0, f(1)=g(1)=1, f(\top)=\top_{1}, g(\top)=\top_{2}$.

By applying $P^{\prime}:$ Pos ${ }^{\text {op }} \rightarrow$ DL to the above coreflexive inserter in Pos we obtain a reflexive coinserter in $\mathrm{DL}$, which can either be checked directly or by appealing to Proposition 6.15 below. Observe that $P^{\prime} e: P^{\prime} D 2 \rightarrow P^{\prime} X$ is the embedding of the four-element Boolean algebra into the distributive lattice

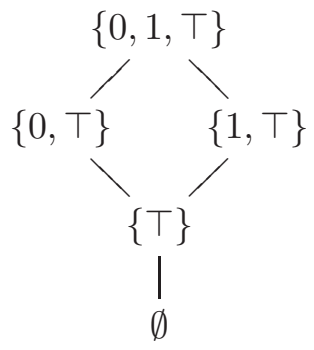

Then it is easy to see that $\bar{L}^{\prime} P^{\prime} e: \bar{L}^{\prime} P^{\prime} X \rightarrow \bar{L}^{\prime} P^{\prime} D 2$ fails to be surjective (since $\bar{L}^{\prime} P^{\prime} D 2$ has four elements and $\bar{L}^{\prime} P^{\prime} X$ only two elements). Hence $\bar{L}^{\prime} P^{\prime} e$ is not the coinserter in DL of the parallel pair $\bar{L}^{\prime} P^{\prime} f, \bar{L}^{\prime} P^{\prime} g$.

Although the locally monotone functor $\bar{L}^{\prime}=W K$ fails to preserve reflexive coinserters, it is however finitary, being a composite of such. Indeed, $W$ is left adjoint, while for $K$ notice the following: $K$ is a right adjoint functor between locally finitely presentable categories, whose left adjoint $W$ preserves finitely presentable objects (see paragraph (2) in the proof of [4, Theorem 1.66]).

The next paragraph will investigate when the functor given by $\bar{L}^{\prime} A=P^{\prime} T^{\prime \text { op }} S^{\prime} A$ on finitely presentable $A$ is not only finitary but also preserves sifted colimits. 
6.F. The case of $\mathbf{L}^{\prime}=P^{\prime} T^{\text {op }} S^{\prime}$ on discretely finitely generated free algebras. Recall that in Section 3.E we have denoted by $\mathbf{J}: \mathrm{BA}_{f f} \rightarrow \mathrm{BA}$ and $\mathbf{J}^{\prime}: \mathrm{DL}_{f f} \rightarrow \mathrm{DL}$ the inclusion functors of the full subcategories spanned by the algebras which are free on finite discrete posets.

Definition 6.7. Let $T^{\prime}$ be an endofunctor of Pos. We define the logic for $T^{\prime}$ to be the pair $\left(\mathbf{L}^{\prime}, \boldsymbol{\delta}^{\prime}\right)$, where:

(1) $\mathbf{L}^{\prime}: \mathrm{DL} \rightarrow \mathrm{DL}$ is an endofunctor of Pos preserving sifted colimits, whose restriction to discretely finitely generated free distributive lattices is $P^{\prime} T^{\prime \text { op }} S^{\prime} \mathbf{J}^{\prime}$, that is, $\mathbf{L}^{\prime}=$ $\operatorname{Lan}_{\mathbf{J}^{\prime}}\left(P^{\prime} T^{\prime \mathrm{op}} S^{\prime} \mathbf{J}^{\prime}\right)$.

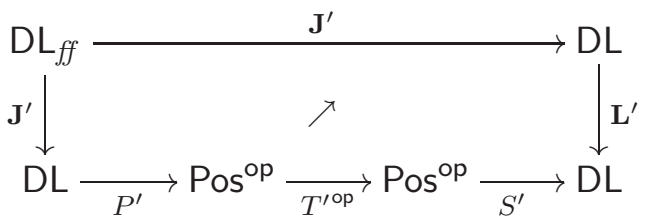

(2) $\boldsymbol{\delta}^{\prime}: \mathbf{L}^{\prime} P^{\prime} \rightarrow P^{\prime} T^{\prime \text { op }}$ is the pasting composite

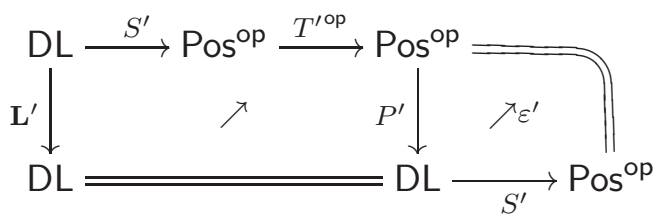

that is, the adjoint transpose of the natural transformation $\mathbf{L}^{\prime} \rightarrow P^{\prime} T^{\prime \text { op }} S^{\prime}$, which in turn is given by the universal property of the left Kan extension $\mathbf{L}^{\prime}$.

Remark 6.8. By Corollary 3.11, $\mathbf{L}^{\prime}$ defined above preserves sifted colimits. Thus, by Theorem 5.8, the functor $\mathbf{L}^{\prime}$ admits an equational presentation by monotone operations, which subsequently gives rise to a positive modal logic concretely given in terms of modal operators and axioms.

Recall from the previous section that we have introduced the functor $\bar{L}^{\prime}$, which on finitely presentable distributive lattices is $P^{\prime} T^{\prime \text { op }} S^{\prime}$, while now we have defined $\mathbf{L}^{\prime}=P^{\prime} T^{\prime o p} S^{\prime}$ only on discretely finitely generated free distributive lattices. The next theorem will provide sufficient conditions for these two functors $\bar{L}^{\prime}$ and $\mathbf{L}^{\prime}$ to coincide.

First note that the restrictions of $\bar{L}^{\prime}$ and $\mathbf{L}^{\prime}$ to $\mathrm{DL}_{f f}$ coincide by definition with $P^{\prime} T^{\prime \mathrm{op}} S^{\prime} \mathbf{J}^{\prime}$. Abbreviate as in (6.4) $\bar{L}_{f}^{\prime}=\bar{L}^{\prime} J^{\prime}$ and $\mathbf{L}_{f f}^{\prime}=\mathbf{L}^{\prime} \mathbf{J}^{\prime}$. Also observe that due to [33, Theorem 4.47], the functor $\mathbf{L}^{\prime}=\operatorname{Lan}_{\mathbf{J}^{\prime}} \mathbf{L}_{f f}^{\prime}$ can equally be expressed as the iterated Kan extension

$$
\operatorname{Lan}_{J^{\prime}}\left(\operatorname{Lan}_{J^{\prime \prime}} \mathbf{L}_{f f}^{\prime}\right)
$$


Because $\bar{L}^{\prime}=\operatorname{Lan}_{J^{\prime}} \bar{L}_{f}^{\prime}$, it will be then enough to show that $\bar{L}_{f}^{\prime}=\operatorname{Lan}_{J^{\prime \prime}} \mathbf{L}_{f f}^{\prime}$, or, in other words, that $P^{\prime} T^{\text {op }} S^{\prime} J^{\prime}$ is the left Kan extension of its restriction to $J^{\prime \prime}$.

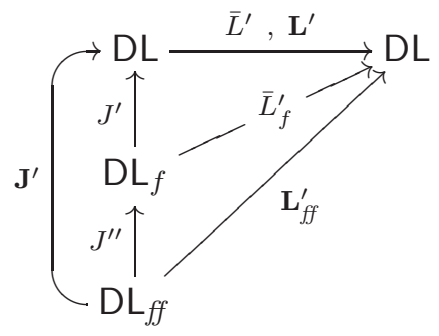

This will follow once we have shown that $J^{\prime \prime}$ is dense and that $P^{\prime} T^{\prime \text { op }} S^{\prime} J^{\prime}$ preserves the colimits of its density presentation.

Theorem 6.9. The inclusion $J^{\prime \prime}: \mathrm{DL}_{f f} \rightarrow \mathrm{DL}_{f}$ from discretely finitely generated free distributive lattices to finitely presentable distributive lattices is dense. A density presentation is given by reflexive coinserters.

Proof. According to the definition of density presentation [33, Theorem 5.19], we have to show that reflexive coinserters exist in $\mathrm{DL}_{f}$, that they are $J^{\prime \prime}$-absolute, and that every object in $\mathrm{DL}_{f}$ can be constructed from objects in $\mathrm{DL}_{f f}$ using reflexive coinserters.

First, note that $\mathrm{DL}_{f}$ has reflexive coinserters since a distributive lattice is in $\mathrm{DL}_{f}$ if and only if it is finite, and since a coinserter of finite distributive lattices is finite.

Second, to say that a reflexive coinserter is $J^{\prime \prime}$-absolute is to say that it is preserved by $\mathrm{DL}\left(F^{\prime} D n,-\right)$ for all finite sets $n$. But $\mathrm{DL}\left(F^{\prime} D n,-\right) \cong \operatorname{Pos}\left(D n, U^{\prime}-\right)$, which preserves reflexive coinserters since finite products preserve reflexive coinserters, and so does $U^{\prime}$.

Finally, we have to prove that every finite distributive lattice is in the closure of $\mathrm{DL}_{f f}$ under reflexive coinserters. Notice that every distributive lattice $F^{\prime} X$ which is free over a finite poset $X$ is such a reflexive coinserter. This is immediate from $F^{\prime}$ preserving colimits and from Proposition 3.4 which presents any poset $X$ as a reflexive coinserter of discrete posets. It remains to show that every finite distributive lattice $A$ is a reflexive coinserter of finitely generated free ones. Let $A$ be a finite distributive lattice and consider the counit

$$
\varepsilon_{A}: F^{\prime} U^{\prime} A \rightarrow A
$$

of the Pos-enriched adjunction $F^{\prime} \dashv U^{\prime}: \mathrm{DL} \rightarrow$ Pos. Since $\varepsilon_{A}$ is surjective, it is a coinserter of some pair

$$
A^{\prime} \stackrel{l^{\prime}}{\underset{r^{\prime}}{\rightleftarrows}} F^{\prime} U^{\prime} A
$$

(by factoring the pair through its image, we can assume without loss of generality that $A^{\prime}$ is finite). Now pre-compose this pair with $\varepsilon_{A^{\prime}}: F^{\prime} U^{\prime} A^{\prime} \rightarrow A^{\prime}$ to obtain

$$
F^{\prime} U^{\prime} A^{\prime} \underset{r^{\prime} \circ \varepsilon_{A^{\prime}}}{\stackrel{l^{\prime} \circ \varepsilon_{A^{\prime}}}{\longrightarrow}} F^{\prime} U^{\prime} A \stackrel{\varepsilon_{A}}{\longrightarrow} A
$$

which is again a coinserter of free distributive lattices on finite posets since $\varepsilon_{A^{\prime}}$ is surjective. Notice that we can always turn the coinserter (6.8) into a reflexive one, namely

$$
F^{\prime} U^{\prime} A+F^{\prime} U^{\prime} A^{\prime} \underset{r}{\stackrel{l}{\rightleftarrows}} F^{\prime} U^{\prime} A \stackrel{\varepsilon_{A}}{\longrightarrow} A
$$


where the parallel arrows $l=\left[\operatorname{id}_{F^{\prime} U^{\prime} A}, l^{\prime} \circ \varepsilon_{A^{\prime}}\right]$ and $r=\left[\operatorname{id}_{F^{\prime} U^{\prime} A}, r^{\prime} \circ \varepsilon_{A^{\prime}}\right]$ are given by the universal property of the coproduct, with common splitting in : $F^{\prime} U^{\prime} A \rightarrow F^{\prime} U^{\prime} A+F^{\prime} U^{\prime} A^{\prime}$ provided by the canonical injection into the coproduct, as the diagram below indicates:

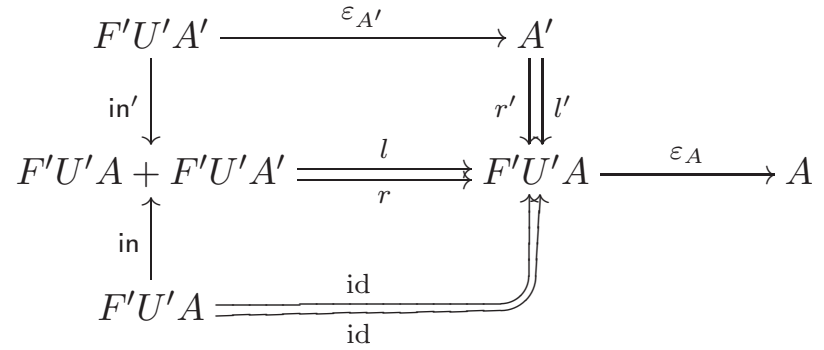

To see that (6.9) is indeed a coinserter, notice first that the inequality $\varepsilon_{A} \circ l \leq \varepsilon_{A} \circ r$ follows from $\varepsilon_{A} \circ l^{\prime} \leq \varepsilon_{A} \circ r^{\prime}$ and from the 2-dimensional property of the Pos-enriched coproduct.

Next, let $h: F^{\prime} U^{\prime} A \rightarrow B$ be an arrow with $h \circ l \leq h \circ r$. It follows that

$$
h \circ l^{\prime} \circ \varepsilon_{A^{\prime}}=h \circ l \circ \text { in } \leq h \circ r \circ \text { in }=h \circ r^{\prime} \circ \varepsilon_{A^{\prime}}
$$

and since $\varepsilon_{A^{\prime}}$ is onto, we obtain

$$
h \circ l^{\prime} \leq h \circ r^{\prime}
$$

But $\varepsilon_{A}$ is the coinserter of the pair $l^{\prime}, r^{\prime}$, therefore there is an arrow $k: A \rightarrow B$ such that $h=k \circ \varepsilon_{A}$. Since $\varepsilon_{A}$ is onto, $k$ is unique. We have therefore shown that in (6.9), the morphism $\varepsilon_{A}$ is the reflexive coinserter of the pair $l, r$.

Using now in (6.9) that

$$
F^{\prime} U^{\prime} A+F^{\prime} U^{\prime} A^{\prime} \cong F^{\prime}\left(U^{\prime} A+U^{\prime} A^{\prime}\right)
$$

we see that the finite distributive lattice $A$ can be obtained as a reflexive coinserter of distributive lattices that are free over finite posets. Since we have already established that the latter are in the closure of $\mathrm{DL}_{f f}$ under reflexive coinserters, the proof is finished.

Remark 6.10. Since the class of reflexive coinserters is definable by a weight, the theorem also shows that $\mathrm{DL}_{f}$ is the free cocompletion of $\mathrm{DL}_{f f}$ by reflexive coinserters, see Proposition [38, Proposition 4.1]. Note that the only particular property of the ordered variety DL used in the proof is that DL is locally finite, that is, that finitely generated algebras are finite.

It follows from the theorem that a functor with domain $\mathrm{DL}_{f}$ is the left Kan extension of its restriction along $J^{\prime \prime}: \mathrm{DL}_{f f} \rightarrow \mathrm{DL}_{f}$ if it preserves reflexive coinserters. Being interested in the composite functor $P^{\prime} T^{\prime \mathrm{oP}} S^{\prime} J^{\prime}: \mathrm{DL}_{f} \rightarrow \mathrm{DL}$, our next step is to establish that $P^{\prime}:$ Pos ${ }^{\mathrm{op}} \rightarrow \mathrm{DL}$ preserves reflexive coinserters. We split this in several lemmas.

Lemma 6.11. Let $e: E \rightarrow X$ be an embedding (i.e., a monotone and order-reflecting map) of posets. Then $[e, 2]$ has a right inverse.

Proof. Remember that any poset can be seen as a category enriched over the two-elements poset $\mathbb{2}$, and any monotone map $e: E \rightarrow X$ as a $\mathcal{2}$-enriched functor.

Pre-composition with $e$ gives a monotone map (hence, a 2 -functor) $[e, \mathcal{L}]:[X, \mathcal{2}] \rightarrow$ $[E, \mathcal{2}]$ which always has a left adjoint $\exists_{e}:[E, \mathbb{2}] \rightarrow[X, \mathcal{2}]$, given by left Kan extension along $e$ (but also a right adjoint $\forall_{e}:[E, \mathbb{Z}] \rightarrow[X, \mathbb{2}]$, provided by the right Kan extension along $e$ ). 
Explicitly, for a monotone map $f: E \rightarrow \mathcal{2}$, the left adjoint acts as follows: $\exists_{e}(f): X \rightarrow \mathcal{L}$ is the monotone map given by

$$
\exists_{e}(f)(x)=1 \Longleftrightarrow \exists a \in E \cdot(f(a)=1 \wedge e(a) \leq x)
$$

for any $x \in X$.

The unit and counit of the adjunction $\exists_{e} \dashv[e, \mathcal{2}]$ are the inequalities

$$
\begin{aligned}
& \operatorname{id}_{[E, \mathbb{L}]} \leq[e, \mathcal{L}] \circ \exists_{e} \\
& \exists_{e} \circ[e, \mathcal{L}] \leq \operatorname{id}_{[X, \mathbb{L}]}
\end{aligned}
$$

By hypothesis, $e$ is an embedding. This means precisely that $e$ is fully faithful as a $\mathbb{2}$ enriched functor. Therefore, the unit $f \leq[e, \mathcal{2}] \circ \exists_{e}(f)$ of the left Kan extension $\exists_{e}(f)$ of $f: E \rightarrow \mathcal{Z}$ along $e$ is an isomorphism [33, Proposition 4.23]. But since $\mathcal{Z}$ is a poset, isomorphism means equality and we obtain

$$
\operatorname{id}_{[E, \mathbb{L}]}=[e, \mathcal{L}] \circ \exists_{e} .
$$

improving (6.10).

Remember the notion of an exact square from Definition 4.9, We shall give now an equivalent formulation:

Lemma 6.12 (Beck-Chevalley Property). The diagram (4.6) exhibits an exact square if and only if $[g, \mathcal{2}] \circ \exists_{f}=\exists_{\alpha} \circ[\beta, \mathcal{2}]$. That is

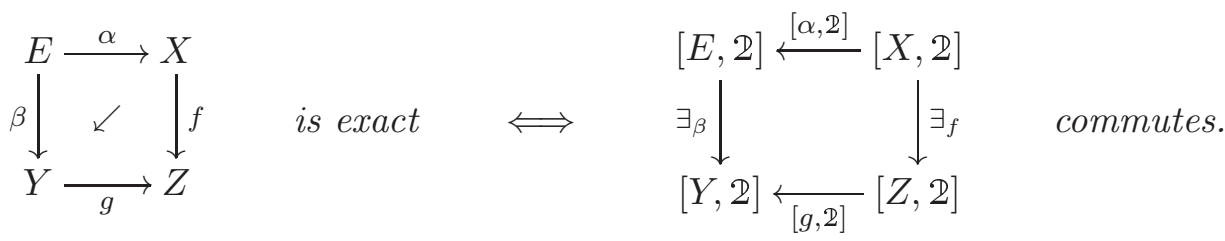

Proof. It follows easily by direct computation.

As in the proof of subsequent Proposition 6.15 we shall encounter split coinserters - the ordered analogue of split coequalizers [47, § VI.6], we provide below the precise definition:

Definition 6.13. In a Pos-category, the diagram below is called a split coinserter if it satisfies the following equations and inequations:

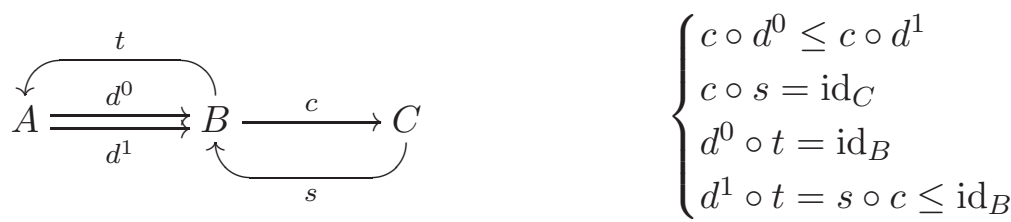

\section{Proposition 6.14.}

(1) A split coinserter is a coinserter.

(2) Split coinserters are absolute: they are preserved by all locally monotone functors.

Proof. The second statement is immediate. To show the first one, let $h: B \rightarrow D$ be an arrow such that $h \circ d^{0} \leq h \circ d^{1}$. Define $k: C \rightarrow D$ by $k=h \circ s$. We have

$$
k \circ c=h \circ s \circ c \leq h
$$


and

$$
k \circ c=h \circ s \circ c=h \circ d^{1} \circ t \geq h \circ d^{0} \circ t=h
$$

Therefore $k \circ c=h$. And $k$ is unique with this property since $c$ is (split) epi.

We have now all ingredients to come back to the question of $P^{\prime}$ preserving reflexive coinserters.

Proposition 6.15. The functor $P^{\prime}:$ Pos $^{\mathrm{op}} \rightarrow$ DL preserves reflexive coinserters.

Proof. Notice that $U^{\prime} P^{\prime}=[-, 2]$ and that $U^{\prime}$ is monadic. Since DL is an ordered variety, it has all (sifted) colimits, in particular reflexive coinserters, and $U^{\prime}$ creates them. Thus it is enough to show that $[-, 2]:$ Pos $^{\mathrm{op}} \rightarrow$ Pos preserves reflexive coinserters.

But reflexive coinserters in Pos ${ }^{\text {op }}$ are coreflexive inserters in Pos; consider therefore two monotone maps with common left inverse in Pos

$$
\stackrel{i}{X \underset{g}{\rightleftharpoons} Y}
$$

The inserter of the above data is realized as the poset ins $(f, g)=\{x \in X \mid f(x) \leq g(x)\}$ with the order inherited from $X$, together with the inclusion map ins $(f, g) \stackrel{e}{\rightarrow} X$. In particular, the diagram below is an exact square:

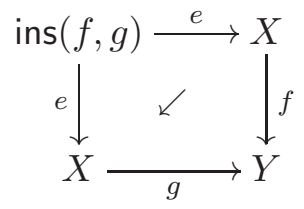

By Lemma 6.12, we obtain $[g, \mathcal{2}] \circ \exists_{f}=\exists_{e} \circ[e, \mathcal{2}]$. Additionally, as both $e$ and $f$ are embeddings (recall that the inserter pair $f, g$ was assumed to have a common left inverse), $[e, \mathcal{L}] \circ \exists_{e}=\operatorname{id}_{[\operatorname{ins}(f, g), \mathcal{L}]}$ and $[f, \mathcal{2}] \circ \exists_{f}=\operatorname{id}_{[X, \mathcal{L}]}$ by Lemma 6.11]

That is, by applying $[-, 2]$ to the diagram (6.14) augmented by ins $(f, g) \stackrel{e}{\rightarrow} X$ and $\exists_{e}, \exists_{f}$, we obtain the split coinserter

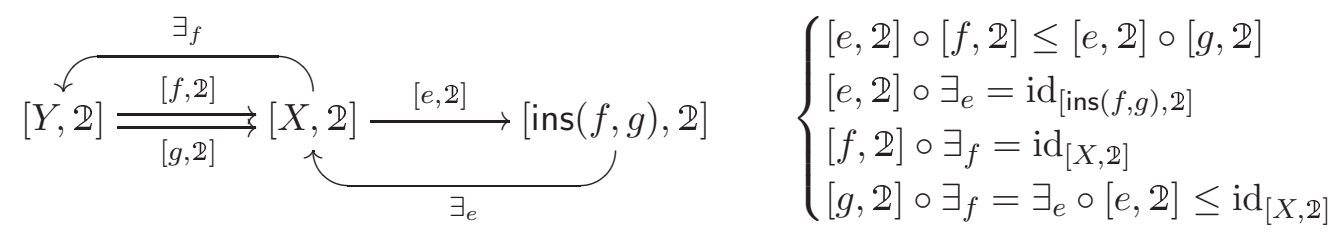

where the first inequation is due to $[-, 2]$ being a locally monotone functor, the next two equations correspond to (6.12), the last equation follows from Lemma 6.12, while the last inequality is due to (6.11). Use then Proposition 6.14 to conclude that $[-, 2]$ maps coreflexive inserters to (split and reflexive) coinserters. 
The following theorem is the technical result around which this section revolves.

Theorem 6.16. Let $\bar{L}=\operatorname{Lan}_{J^{\prime}}\left(P^{\prime} T^{\prime o p} S^{\prime} J^{\prime}\right)$ and $\mathbf{L}=\operatorname{Lan}_{\mathbf{J}^{\prime}}\left(P^{\prime} T^{\prime o p} S^{\prime} \mathbf{J}^{\prime}\right)$. If $T^{\prime}$ sends coreflexive inserters of finite posets into coreflexive inserters, then $\bar{L}^{\prime} \cong \mathbf{L}^{\prime}$.

Proof. Taking up the reasoning preceding (6.7), we need to show that $P^{\prime} T^{\prime \text { op }} S^{\prime} J^{\prime}$ is the left Kan extension of its restriction along $J^{\prime \prime}$. We know that $P^{\prime} T^{\prime \text { op }} S^{\prime} J^{\prime}$ preserves reflexive coinserters of finite distributive lattices. Indeed, $J^{\prime}$ is a completion by filtered colimits and preserves all finite colimits 4 $; S^{\prime}$ as a left adjoint preserves all colimits (hence it maps reflexive coinserters to coreflexive inserters) and maps finite distributive lattices to finite posets; $T^{\prime \text { op }}$ preserves coreflexive inserters of finite posets by hypothesis, and $P^{\prime}$ sends them to reflexive coinserters, due to Proposition 6.15. Our claim now follows from Theorem 6.9 and [33, Theorem 5.29].

As a result about modal logics, the theorem can be reformulated as follows.

Corollary 6.17. Let $T$ be an endofunctor on Set and $T^{\prime}$ a Pos-extension of $T$ which preserves coreflexive inserters. Then $\left(\bar{L}^{\prime}, \bar{\delta}^{\prime}\right)$ and $\left(\mathbf{L}^{\prime}, \boldsymbol{\delta}^{\prime}\right)$ coincide. In particular, it follows from Proposition 6.5 that $\mathbf{L}^{\prime}$ is the maximal positive fragment of $\mathbf{L}$.

The next example shows what can go wrong in case that $T^{\prime}$ does not preserve coreflexive inserters.

Example 6.18. For $T=\mathrm{Id}$, the corresponding finitary logics is $\mathbf{L}=\mathrm{Id}$ on BA, with trivial semantics $\delta: L P \rightarrow P T^{\mathrm{op}}$. It was noticed in Remark 4.2)(2) that the identity functor also admits as extension the discrete connected components functor $T^{\prime}=D C$. But the latter preserves neither embeddings, nor coreflexive inserters. The corresponding logic $\mathbf{L}^{\prime}$ for $T^{\prime}$ is given by the constant functor to the distributive lattice $\mathcal{P}$. Thus the natural transformation $\beta: \mathbf{L}^{\prime} W \rightarrow W \mathbf{L}$ from Definition 6.2 fails to be an isomorphism (it is just the unique morphism from the initial object).

Whereas the associated 'strongly finitary' logic $\mathbf{L}^{\prime}$ of $T^{\prime}=D C$ is just the logic of the constant functor $\mathcal{2}$ (i.e., plain positive propositional logic), the associated 'finitary logic' $\bar{L}^{\prime}$ is given by the functor $W K$ on DL. This can be seen as follows: on finite distributive lattices, $\bar{L}^{\prime}$ is $P^{\prime} D^{\mathrm{op}} C^{\mathrm{op}} S^{\prime} \cong W P C^{\mathrm{op}} S^{\prime} \cong W K P^{\prime} S^{\prime} \cong W K$. As $W K$ is finitary (Example 6.6), it coincides with $\bar{L}^{\prime}$ on all distributive lattices, not just on the finite ones.

The next lemma shows that for a locally monotone functor on Pos, preservation of exact squares entails the condition needed in Theorem 6.16, namely the preservation of coreflexive inserters:

Lemma 6.19. If $T^{\prime}$ is a locally monotone endofunctor on Pos which preserves exact squares, then it preserves embeddings and coreflexive inserters.

Proof. The first assertion follows from the observation 25] that each embedding $e: X \rightarrow Y$ can be realized as an exact square, namely

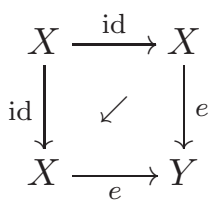


For the second one, let

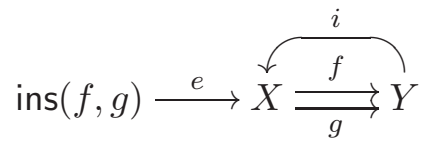

be a coreflexive inserter. In particular,

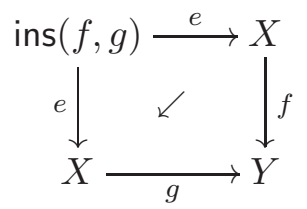

is an exact square as remarked in the proof of Proposition 6.15, thus $T^{\prime}$ maps it to the exact square

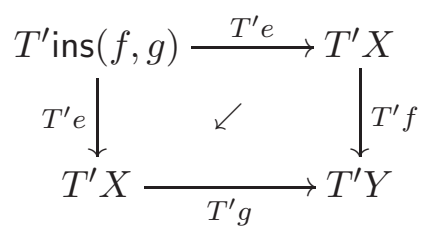

Let now $u: U \rightarrow T^{\prime} X$ a monotone map such that $T^{\prime} f \circ u \leq T^{\prime} g \circ u$. For each $x \in$ $U, T^{\prime} f(u(x)) \leq T^{\prime} g(u(x))$, thus there is some $w \in T^{\prime} \operatorname{ins}(f, g)$ with $u(x) \leq T^{\prime} e(w)$ and $T^{\prime} e(w) \leq u(x)$, that is, $u(x)=T^{\prime} e(w)$. As $T^{\prime} e$ is an embedding, such element $w$ is uniquely determined. Moreover, the assignment $x \mapsto w$ is monotone, as if $x_{1} \leq x_{2}$, then $T^{\prime} e\left(w_{1}\right)=$ $u\left(x_{1}\right) \leq u\left(x_{2}\right)=T^{\prime} e\left(w_{2}\right)$ and $T^{\prime} e$ is again an embedding as shown earlier, hence $w_{1} \leq w_{2}$. This covers the 1-dimensional aspect of inserters. For the rest, use one more time that $T^{\prime} e$ is an embedding.

As a consequence of all the results of this section and of Theorem 4.10, we obtain our main theorem on positive modal logic.

Theorem 6.20. Let $T:$ Set $\rightarrow$ Set be a weak-pullback preserving functor and $T^{\prime}:$ Pos $\rightarrow$ Pos its posetification. Let $(\mathbf{L}, \boldsymbol{\delta})$ and $\left(\mathbf{L}^{\prime}, \boldsymbol{\delta}^{\prime}\right)$ be the associated logics of $T$ and $T^{\prime}$, that is $\mathbf{L}=\operatorname{Lan}_{\mathbf{J}}\left(P T^{\mathrm{op}} S \mathbf{J}\right)$ and $\mathbf{L}^{\prime}=\operatorname{Lan}_{\mathbf{J}^{\prime}}\left(P^{\prime} T^{\text {op }} S^{\prime} \mathbf{J}^{\prime}\right)$. Then $\left(\mathbf{L}^{\prime}, \boldsymbol{\delta}^{\prime}\right)$ is the maximal positive fragment of $(\mathbf{L}, \boldsymbol{\delta})$.

Corollary 6.21. Under the hypotheses of Theorem 6.20, the adjunction $W \dashv K: \mathrm{DL} \rightarrow \mathrm{BA}$ lifts to an adjunction $\widetilde{W} \vdash \widetilde{K}: \operatorname{Alg}\left(\mathbf{L}^{\prime}\right) \rightarrow \operatorname{Alg}(\mathbf{L})$.

Proof. Recall from Definition 6.2 that the statement of Theorem 6.20 implies that there is a natural isomorphism

$$
\boldsymbol{\beta}: \mathbf{L}^{\prime} W \rightarrow W \mathbf{L} .
$$

By Remark 6.1, and by standard doctrinal adjunction [32, we know then that the adjunction $W \dashv K: \mathrm{DL} \rightarrow \mathrm{BA}$ lifts to an adjunction between the corresponding categories of algebras, where the lifting $\widetilde{W}$ of $W$ maps an $\mathbf{L}$-algebra

$$
\mathbf{L} B \stackrel{b}{\longrightarrow} B
$$

to the $\mathbf{L}^{\prime}$-algebra

$$
\mathbf{L}^{\prime} W B \stackrel{\boldsymbol{\beta}}{\longrightarrow} W \mathbf{L} B \stackrel{W b}{\longrightarrow} W B
$$


The above corollary shows in particular that $\operatorname{Alg}(\mathbf{L})$ is a full coreflective subcategory of $\operatorname{Alg}\left(\mathbf{L}^{\prime}\right)$ (using that $\tilde{W}$ is fully-faithful, property inherited from $W$ ), and that $\widetilde{W}$ preserves initial algebras. In other words, the Lindenbaum algebra for $\mathbf{L}$ is the same as the one for $\mathbf{L}^{\prime}$ (the analogous statement for free algebras only holds if the generating atomic propositions are closed under complement).

Remark 6.22. Our introductory example of positive modal logic is now regained as an instance of this theorem. It can also easily be adapted to Kripke polynomial functors. More interesting are the cases of probability distribution functor and of multiset functor. We know from the theorem above that they have maximal positive fragments, but their explicit description still needs to be worked out.

To see how Dunn's completeness result can be obtained in our setting, we first remark that, from an algebraic point of view, completeness follows from Theorem 6.20. Indeed, let $I, I^{\prime}$ be the initial algebras (i.e., the Lindenbaum algebras) for the functors $\mathbf{L}$ and $\mathbf{L}^{\prime}$, respectively. Then the induced arrow $I^{\prime} \rightarrow W I$ is an isomorphism. Hence, if two elements (i.e., formulas) are equal in $I$ they also must be equal in $I^{\prime}$, which implies, by completeness of equational logic, that every proof with $\mathbf{L}$-axioms can be imitated with $\mathbf{L}^{\prime}$-axioms. Here, the terminology of axioms refers to presentations of the functors in the sense of Definitions 5.1 and 5.5 .

From the point of view of Kripke semantics, completeness means that if two formulas have the same semantics, then they are equal. In other words, recalling Section 2.E, with $(X, \xi)$ ranging over all coalgebras, the family $\llbracket \cdot \rrbracket_{(X, \xi)}: I \rightarrow P X$, or rather $\llbracket \cdot \rrbracket_{(X, \xi)}: I^{\prime} \rightarrow P^{\prime} X$ must be jointly injective. As in [40], this follows from the one-step semantics $\delta^{\prime}: \mathbf{L}^{\prime} P^{\prime} \rightarrow P^{\prime} T^{\prime}$ being injective. We sketch below (Paragraphs $\mathbf{A}$ D) some of the details.

A. First, we show that for all posetifications $T^{\prime}:$ Pos $\rightarrow$ Pos and $X^{\prime}$ in Pos the one-step semantics $\delta_{X^{\prime}}^{\prime}: \mathbf{L}^{\prime} P^{\prime} X^{\prime} \rightarrow P^{\prime} T^{\prime} X^{\prime}$ is injective. Up to replacing $\delta, L, P, T$ by $\delta^{\prime}, \mathbf{L}^{\prime}, P^{\prime}, T^{\prime}$, the proof is verbatim the same as in [42, Lemma 6.12], using Theorem 6.16 in order to know that $\mathbf{L}^{\prime} P^{\prime} X^{\prime}$ is given as a filtered colimit as in [42, (44)] and using Proposition 4.15] in order to know that the posetification $T^{\prime}$ preserves surjections.

B. Next, in analogy with [42, Lemma 6.14], we show that $\mathbf{L}^{\prime}$ preserves injections. Let us emphasize that whereas all functors $\mathrm{BA} \rightarrow \mathrm{BA}$ with a presentation by operations and equations preserve injections, the same is not true for functors $\mathrm{DL} \rightarrow \mathrm{DL}$, see [48, Section 5.3].

Proposition 6.23. Assume that the hypotheses of Theorem 6.20 hold. Then $\mathbf{L}^{\prime}$ preserves monomorphisms of distributive lattices.

Proof. We shall proceed in two steps:

(1) $\mathbf{L}^{\prime}$ preserves monomorphisms between finite distributive lattices. This can be seen as follows: recall that on finite distributive lattices, $\mathbf{L}^{\prime}$ is $P^{\prime} T^{\prime \mathrm{op}} S^{\prime}$ (Theorem 6.16), and let $m: A \rightarrow B$ be an injective morphism between two finite distributive lattices. According to [60, Theorem 4.2], $m$ is the coreflexive inserter of its co-comma square:

$$
A \stackrel{m}{\longrightarrow} B \stackrel{p}{\underset{q}{\longrightarrow}} C
$$


Notice that $C$ is also finite. But on finite distributive lattices, $S^{\prime}$ is an equivalence. Therefore $S^{\prime} m$ is the reflexive coinserter of its comma object $\left(S^{\prime} p, S^{\prime} q\right)$, in particular, a monotone surjection. By Proposition 4.15, $T^{\prime} S^{\prime} m$ will also be a surjective monotone map between posets, thus a monomorphism in Pos ${ }^{\mathrm{op}}$. As $P^{\prime}$ is right adjoint, it sends $T^{\prime \mathrm{op}} S^{\prime} m$ to a monomorphism in DL. Thus $\mathbf{L}^{\prime} m \cong P^{\prime} T^{\prime \mathrm{op}} S^{\prime} m$ is again a monomorphism.

(2) $\mathbf{L}^{\prime}$ preserves arbitrary monomorphisms. Let $m: A \rightarrow B$ be a monomorphism of distributive lattices. But DL is a locally finitely presentable category, the finitely presentable objects being precisely the finite ones. Thus [13, Corollary 4.3] applies to conclude that $m$ can be expressed as a filtered colimit of monomorphisms between finite distributive lattices. We can now use step (1) and that $\mathbf{L}^{\prime}$ preserves filtered colimits, to obtain that $\mathbf{L}^{\prime} m$ is again a monomorphism (see also [4, Corollary 1.60]).

Remark 6.24. The above proposition shows that the modal logic given by any presentation of $\mathbf{L}^{\prime}$ has the bounded proof property in the sense of [11].

C. We can now continue the reasoning begun in Paragraphs $\mathrm{A} B$, Using Proposition 6.23, we are able to repeat the proof of [42, Theorem 6.15], showing by induction that the $n$-step semantics

$$
\left(\mathbf{L}^{\prime}\right)^{n} \mathcal{Z} \rightarrow P^{\prime}\left(T^{\prime}\right)^{n} 1
$$

is injective for all $n \in \mathbb{N}$, which implies completeness with respect to the Kripke semantics given by $T^{\prime}$-coalgebras.

D. Finally, since $T^{\prime}$ is an extension of $T$, we have $T^{\prime} D \cong D T$, which implies together with $P^{\prime} D \cong W P$ that $P^{\prime}\left(T^{\prime}\right)^{n} 1 \cong W P T^{n} 1$, so that (6.16) also gives completeness with respect to the Kripke semantics given by $T$-coalgebras.

Remark 6.25. As in Corollary 6.21, or rather dually, the fact that we have $T^{\prime} D \cong D T$ means that the adjunction $C \dashv D:$ Set $\rightarrow$ Pos lifts to an adjunction $\widetilde{C} \dashv \widetilde{D}: \operatorname{Coalg}(T) \rightarrow$ Coalg $\left(T^{\prime}\right)$ so that $\operatorname{Coalg}(T)$ is a full reflective subcategory of Coalg $\left(T^{\prime}\right)$. In particular, $\widetilde{D}$ preserves limits and therefore 'behaviours' as given by final coalgebras or the final sequence of $T$.

\section{Monotone PRedicate Liftings}

We show that the logic of the posetification $T^{\prime}$ of $T$ coincides with the logic of all monotone predicate liftings of $T$.

Recall from [50, 53] that a predicate lifting of arity $n$ for $T$ is a natural transformation

$$
\varnothing: \operatorname{Set}\left(-, 2^{n}\right) \rightarrow \operatorname{Set}(T-, 2)
$$

Using the (ordinary!) adjunction $D \dashv V:$ Pos $\rightarrow$ Set, a predicate lifting can be described as a natural transformation

$$
\bigcirc^{\prime}: \operatorname{Pos}(D-,[D n, 2]) \rightarrow \operatorname{Pos}(D T-, 2)
$$

It is called monotone if each component is monotone (as a map between hom-posets). By Yoneda lemma, one can also identify a predicate lifting with a map $\varnothing: T\left(2^{n}\right) \rightarrow 2$. Then the above simply says that $\varnothing$ is monotone if for all $\overline{a_{1}} \leq \overline{a_{2}}: D X \rightarrow[D n, 2]$, we have that $\overline{\nabla \circ T a_{1}} \leq \overline{\nabla \circ T a_{2}}$, where $\bar{f}: D X \rightarrow Y$ denotes the adjoint transpose of $f: X \rightarrow V Y$. 
Consider now a locally monotone Pos-functor $T^{\prime}$ and a finite poset $p$. By mimicking the above, we define a predicate lifting for $T^{\prime}$ of arity $p$ as being a Pos-natural transformation

$$
\nabla^{\prime}: \operatorname{Pos}(-,[p, \mathcal{2}]) \rightarrow \operatorname{Pos}\left(T^{\prime}-, \mathcal{2}\right)
$$

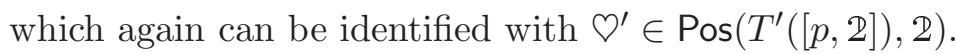

Theorem 7.1. Let $T$ be an endofunctor of Set and $T^{\prime}:$ Pos $\rightarrow$ Pos its posetification. Then there is a bijection between the predicate liftings of $T^{\prime}$ of discrete arity Dn and the monotone predicate liftings of $T$ of arity $n$, for each finite $n$.

Proof. Let $p$ be an arbitrary finite poset. Consider the composition of the two following monomorphisms:

$$
\operatorname{Pos}\left(T^{\prime}([p, \mathcal{2}]), \mathcal{L}\right) \rightarrow \operatorname{Set}\left(V T^{\prime}([p, \mathcal{L}]), V \mathcal{L}\right) \rightarrow \operatorname{Set}(T V([p, \mathcal{2}]), V \mathcal{2})
$$

The first arrow above is monic by faithfulness of $V$. The second one is also, as it is given by pre-composition with the natural epimorphism $\tau: T V \rightarrow V T^{\prime}$ (the mate of the isomorphism $\alpha: D T \rightarrow T^{\prime} D$ under the adjunction $\left.D \dashv V\right)$. The latter is indeed epic because for each poset $X, \tau_{X}$ is exactly the coinserter map $T X_{0} \rightarrow T^{\prime} X$.

In case the arity is discrete as $p=D n$, notice that by $V([D n, \mathcal{2}])=2^{n}$, the right hand side of Equation (17.1) is precisely $\operatorname{Set}\left(T\left(2^{n}\right), 2\right)$. A predicate lifting $\nabla^{\prime} \in \operatorname{Pos}\left(T^{\prime}([D n, \mathcal{2}]), \mathcal{2}\right)$ is then sent to

$$
\odot:=V \varnothing^{\prime} \circ \tau_{[D n, \mathbb{2}]}: T\left(2^{n}\right) \rightarrow 2
$$

Now, for $a: X \rightarrow 2^{n}$, easy diagram chasing shows that

$$
\overline{\varnothing \circ T a}=\varnothing^{\prime} \circ T^{\prime}(\bar{a}) \circ \alpha_{X}
$$

hence the monotonicity of $\varnothing$ follows. Thus the predicate liftings of $T^{\prime}$ of discrete arity are among the monotone predicate liftings for $T$.

To show the inverse correspondence, recall one more time that the posetification $T^{\prime}$ is constructed as a coinserter (Theorem 4.3). Let $\varnothing: T\left(2^{n}\right) \rightarrow 2$ be a predicate lifting for $T$. Then, from the universal property of coinserters, one can easily check that $\bar{\varnothing}: D T\left(2^{n}\right) \rightarrow \mathcal{L}$ factorizes to a predicate lifting for $T^{\prime}$ of discrete arity, $T^{\prime}([D n, \mathcal{2}]) \rightarrow \mathcal{2}$, if and only if $\varnothing$ is monotone in the sense mentioned above. More in detail: let $X_{0}$ be the set $2^{n}=V[D n, \mathcal{2}]$; that is, the underlying set of the poset $[D n, \mathbb{2}]$, and $X_{1}$ the underlying set of the order on $[D n, 2]$, with projections denoted as usual $d^{0}, d^{1}: X_{1} \rightarrow X_{0}$. Then with notations as above,

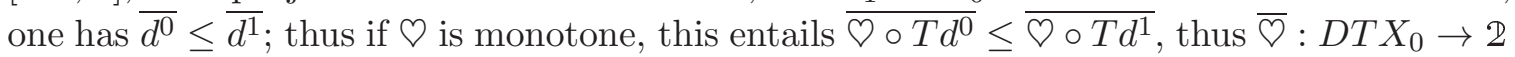
factorizes in Pos to a predicate lifting for $T^{\prime}$ of discrete arity ${\nabla^{\prime}}^{\prime} T^{\prime}[D n, \mathcal{2}] \rightarrow \mathcal{2}$.

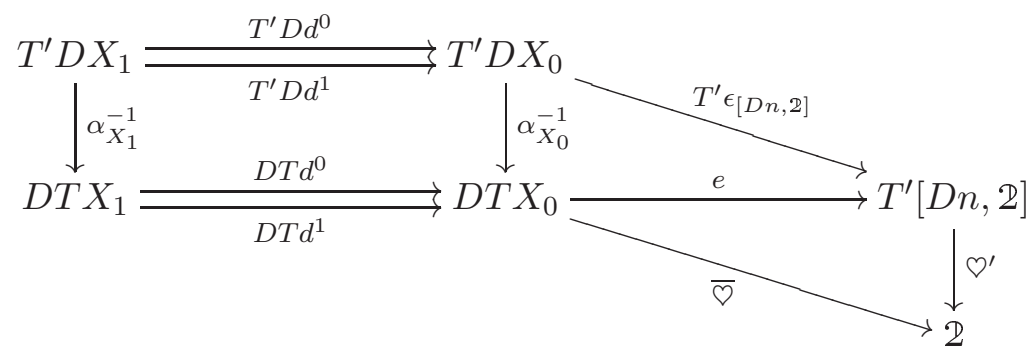

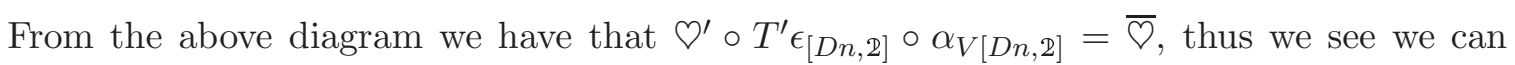
recover the original monotone predicate lifting for $T$ :

$$
V \triangleright^{\prime} \circ \tau_{[D n, \mathbb{L}]}=V \triangleright^{\prime} \circ V T^{\prime} \epsilon_{[D n, \mathbb{L}]} \circ V \alpha_{V[D n, \mathbb{L}]}=V \bar{\varnothing}=\varnothing
$$


Finally, note that we have used the assumption that $T^{\prime}$ is the posetification of $T$ in order to have an extension such that $T V \rightarrow V T^{\prime}$ is epi.

Remark 7.2. The theorem should be seen in the light of [31, Theorem 4.16] saying that for a finitary and embedding preserving functor $T^{\prime}:$ Pos $\rightarrow$ Pos the logic $\mathbf{L}^{\prime}$ of (necessarily monotone) predicate liftings is expressive. We also would like to recall [41, Corollary 6.9] which describes an expressive and monotone subset of all predicates liftings for any finitary weak-pullback preserving $T:$ Set $\rightarrow$ Set.

\section{Conclusions}

In the area of semantics of programming languages one encounters a wide variety of base categories including various metric spaces and various (complete) partial orders. It would be of interest to draw the landscape of these different categories together with a toolkit connecting them. This paper can be seen as a rudimentary effort in this direction. Indeed, we relate systems and their logics across the morphism of connections

$$
\left(S \dashv P: \text { Set }^{\mathrm{op}} \rightarrow \mathrm{BA}\right) \longrightarrow\left(S^{\prime} \dashv P^{\prime}: \mathrm{Pos}^{\mathrm{op}} \rightarrow \mathrm{DL}\right)
$$

Moreover, we transfer functors along this morphism via left Kan-extensions and characterize the functors that arise in that way as those preserving certain classes of colimits. Finally, we show how results about modal logics can be derived from such a framework. It will be interesting to explore whether similar techniques apply to more sophisticated domains than Set and Pos.

\section{REFERENCES}

[1] S. Abramsky, A Cook's tour of the finitary non-well-founded sets. In: S. Artemov et al (eds.), We Will Show Them: Essays in honour of Dov Gabbay, vol. 1, 1-18, College Publications (2005), arXiv 1111.7148

[2] S. Abramsky, Domain theory in logical form. Ann. Pure Appl. Logic 51:1-77 (1991)

[3] S. Abramsky and A. Jung, Domain theory. In: S. Abramsky et al (eds.), Handbook of Logic in Computer Science, vol. 3, 1-168, Clarendon Press (1994)

[4] J. Adámek and J. Rosický, Locally presentable and accessible categories. London Math. Soc. LNS 189, Cambridge Univ. Press (1994)

[5] J. Adámek, J. Rosický and E. Vitale, Algebraic theories. Cambridge Univ. Press (2011)

[6] M. H. Albert and G. M. Kelly, The closure of a class of colimits. J. Pure Appl. Algebra 51:1-17 (1988)

[7] C. Baier, On algorithmic verification methods for probabilistic systems. Habilit. thesis, Fakult. Mathem. Inform., Univ. Mannheim (1998)

[8] A. Balan and A. Kurz, Finitary functors: from Set to Preord and Poset. In: A. Corradini et al (eds.), CALCO 2011, LNCS 6859, 85-99, Springer, Heidelberg (2011)

[9] A. Balan, A. Kurz and J. Velebil, Positive fragments of coalgebraic logics. In: R. Heckel and S. Milius (eds.), CALCO 2013, LNCS 8089, 51-65, Springer, Heidelberg (2013)

[10] M. Barr, Relational algebras. In: S. MacLane et al (eds.), Reports of the Midwest Category Seminar IV, LNM 137, 39-55, Springer, Heidelberg (1970)

[11] N. Bezhanishvili and S. Ghilardi, The bounded proof property via step algebras and step frames. Ann. Pure Appl. Logic 165:1832-1863 (2014)

[12] M. Bílková, A. Kurz, D. Petrişan and J. Velebil, Relation liftings on preorders. In: A. Corradini et al (eds.) CALCO 2011, LNCS 6859, 115-129, Springer, Heidelberg (2011)

[13] G. J. Bird, Limits in 2-categories of locally-presented categories, PhD Thesis, Univ. of Sydney (1984)

[14] G. Birkhoff, Lattice theory. Amer. Math. Soc. (1967)

[15] P. Blackburn, M. de Rijke and Y. Venema, Modal logic. Cambridge Tracts in Theor. Comput. Sci. 53, Cambridge Univ. Press (2002) 
[16] S. L. Bloom, Varieties of ordered algebras. J. Comput. System Sci. 13:200-212 (1976)

[17] S. L. Bloom and J. B. Wright, P-varieties - A signature independent characterization of varieties of ordered algebras. J. Pure Appl. Algebra 29:13-58 (1983)

[18] M. Bonsangue and A. Kurz, Presenting functors by operations and equations. In: L. Aceto and A. Ingólfsdóttir (eds.), FoSSaCS 2006, LNCS 3921, 172-186, Springer (2006)

[19] J. Bourke, Codescent objects in 2-dimensional universal algebra, PhD Thesis, Univ. of Sydney (2010)

[20] A. Carboni and R. Street, Order ideals in categories, Pacific J. Math. 124:275-288 (1986)

[21] A. Carboni, G. M. Kelly and R. J. Wood, A 2-categorical approach to change of base and geometric morphisms I, Cah. Topol. Géom. Différ. Catég. XXXII(1):47-95 (1991)

[22] B. A. Davey and H. A. Priestley, Introduction to lattices and order, 2nd ed. Cambridge Univ. Press $(2002)$

[23] J. M. Dunn, Positive modal logic, Studia Logica 55:301-317 (1995)

[24] R. Goldblatt, Metamathematics of modal logic I, Reports on Mathematical Logic 6:41-78 (1976)

[25] R. Guitart, Rélations et carrés exacts, Ann. Sci. Math. Québec 4:103-125 (1980)

[26] B. Jacobs and A. Sokolova, Exemplaric expressivity of modal logics. J. Log. Comput. 20:1041-1068 (2010)

[27] P. T. Johnstone, Topos Theory. Academic Press, London (1977)

[28] P. T. Johnstone, Stone Spaces. Cambridge Univ. Press (1982)

[29] P. T. Johnstone, Vietoris locales and localic semilattices. In: R. E. Hoffmann and K. H. Hofmann (eds.), Continuous Lattices and their Applications, LNPAM 101, 155-180, Marcel Dekker (1985)

[30] B. Jónsson and A. Tarski, Boolean algebras with operators, Part I. Amer. J. Math. 73:891-939 (1951)

[31] K. Kapulkin, A. Kurz and J. Velebil, Expressiveness of positive coalgebraic logic. Adv. Modal Logic 9:368-385 (2012)

[32] G. M. Kelly, Doctrinal adjunction. In: G. M. Kelly (ed.), Category Seminar, LNM 420, $257-280$. Springer, Berlin Heidelberg (1974)

[33] G. M. Kelly, Basic concepts of enriched category theory. London Math. Soc. LNS 64, Cambridge Univ. Press (1982), Repr. Theory Appl. Categ. 10:1-136 (2005)

[34] G. M. Kelly, Structures defined by finite limits in the enriched context, I. Cah. Topol. Géom. Différ. Catég. XXIII:3-42 (1982)

[35] G. M. Kelly, Elementary observations on 2-categorical limits. Bull. Aust. Math. Soc. 39:301-317 (1989)

[36] G. M. Kelly and S. Lack, Finite-product-preserving functors, Kan extensions and strongly-finitary 2monads. Appl. Categ. Structures 1:85-94 (1993)

[37] G. M. Kelly, S. Lack and R. F. C. Walters, Coinverters and categories of fractions for categories with structure. Appl. Categ. Structures 1:95-102 (1993)

[38] G. M. Kelly, V. Schmitt, Notes on enriched categories with colimits of some class. Theory Appl. Categ. 14:399-423 (2005)

[39] B. Klin, Coalgebraic modal logic beyond sets. Electron. Notes Theor. Comput. Sci. 173:177-201 (2007)

[40] C. Kupke, A. Kurz and D. Pattinson, Algebraic semantics for coalgebraic logics, Electron. Notes Theor. Comput. Sci. 106:219-241 (2004)

[41] A. Kurz and R. Leal, Modalities in the Stone age: A comparison of coalgebraic logics, Theor. Comput. Sci. 430:88-116 (2012)

[42] A. Kurz and D. Petrişan, Presenting functors on many-sorted varieties and applications. Inf. Comput. 208:1421-1446 (2010)

[43] A. Kurz and J. Rosický, Strongly complete logics for coalgebras. Log. Methods Comput. Sci. 8:1-32 (2012)

[44] A. Kurz and J. Velebil, Enriched logical connections. Appl. Categ. Structures 21:349-377 (2013)

[45] A. Kurz and J. Velebil, Quasivarieties and varieties of ordered algebras: Regularity and exactness. To appear in Math. Structures Comput. Sci.

[46] S. Lack and J. Rosický, Notions of Lawvere theory. Appl. Categ. Structures 19:363-391 (2011)

[47] S. Mac Lane, Categories for the working mathematician. GTM 5, Springer (1971)

[48] R. Myers, Rational coalgebraic machines in varieties: languages, completeness and automatic proofs. PhD Thesis, Imperial College London (2011)

[49] A. Palmigiano, A coalgebraic view on positive modal logic. Theoret. Comput. Sci. 327:175-195 (2004)

[50] D. Pattinson, Coalgebraic modal logic: Soundness, completeness and decidability of local consequence. Theoret. Comput. Sci. 309:177-193 (2003) 
[51] E. Robinson, Powerdomains, modalities and the Vietoris monad. Comp. Lab. Tech. Report 98, Univ. of Cambridge (1986)

[52] J. J. M. M. Rutten, Universal coalgebra: a theory of systems. Theor. Comput. Sci. 249:3-80 (2000)

[53] L. Schröder, Expressivity of coalgebraic modal logic: The limits and beyond. In: V. Sassone (ed.), FoSSaCS 2005, LNCS 3441, 440-454, Springer, Berlin (2005)

[54] A. Thijs, Simulation and fixed point semantics. PhD Thesis, Univ. of Groningen (1996)

[55] V. Trnková, Some properties of set functors. Comment. Math. Univ. Carolin. 10(2):323-352 (1969)

[56] V. Trnková, Relational automata in a category and their languages. In: M. Karpinski (ed.), Fundamentals of Computation Theory, LNCS 56, 340-355, Springer, Berlin Heidelberg (1977)

[57] J. Velebil and A. Kurz, Equational presentations of functors and monads. Math. Structures Comput. Sci. 21:363-381 (2011)

[58] E. P. de Vink and J. J. M. M. Rutten, Bisimulation for probabilistic transition systems: A coalgebraic approach. Theoret. Comput. Sci. 221:271-293 (1999)

[59] G. Winskel, On powerdomains and modality. Theoret. Comput. Sci. 36:127-137 (1985)

[60] M. W. Zawadowski, Descent and duality. Ann. Pure Appl. Logic 71(2):131-188 (1995) 